\title{
Local Manifestations of Cometary Activity
}

\author{
Jean-Baptiste Vincent ${ }^{1}$ D - Tony Farnham ${ }^{2}$ - Ekkehard Kührt ${ }^{1}$ - Yuri Skorov ${ }^{3}$. \\ Raphael Marschall $^{4}$ - Nilda Oklay ${ }^{1}$ - Mohamed Ramy El-Maarry ${ }^{5}$. \\ Horst Uwe Keller ${ }^{1,6}$
}

\begin{abstract}
Comets are made of volatile and refractory material and naturally experience various degrees of sublimation as they orbit around the Sun. This gas release, accompanied by dust, represents what is traditionally described as activity. Although the basic principles are well established, most details remain elusive, especially regarding the mechanisms by which dust is detached from the surface and subsequently accelerated by the gas flows surrounding the nucleus.

During its 2 years rendez-vous with comet 67P/Churyumov-Gerasimenko, ESA's Rosetta has observed cometary activity with unprecedented details, in both the inbound and outbound legs of the comet's orbit. This trove of data provides a solid ground on which new models of activity can be built. In this chapter, we review how activity manifests at close distance from the surface, establish a nomenclature for the different types of observed features, discuss how activity is at the same time transforming and being shaped by the topography, and finally address several potential mechanisms.
\end{abstract}

Keywords Comets $\cdot$ Activity $\cdot$ Rosetta

Comets: Post 67P/Churyumov-Gerasimenko Perspectives

Edited by Nicolas Thomas, Björn Davidsson, Laurent Jorda, Ekkehard Kührt, Raphael Marschall, Colin Snodgrass and Rafael Rodrigo

J.-B. Vincent

jean-baptiste.vincent@dlr.de

1 DLR Institute of Planetary Research, Rutherfordstrasse, 2, 12489 Berlin, Germany

2 Department of Astronomy, University of Maryland, College Park, MD 20742, USA

3 Institut für Geophysik und extraterrestrische Physik, Technische Universität Braunschweig, Mendelssohnstr. 3, 38106 Braunschweig, Germany

4 Physikalisches Institut, University of Bern, Sidlerstr. 5, 3012 Bern, Switzerland

5 Birkbeck College, University of London, WC1E 7HX, London, UK

6 Institut fur Geophysik und extraterrestrische Physik, TU Braunschweig, 38106 Braunschweig, Germany 

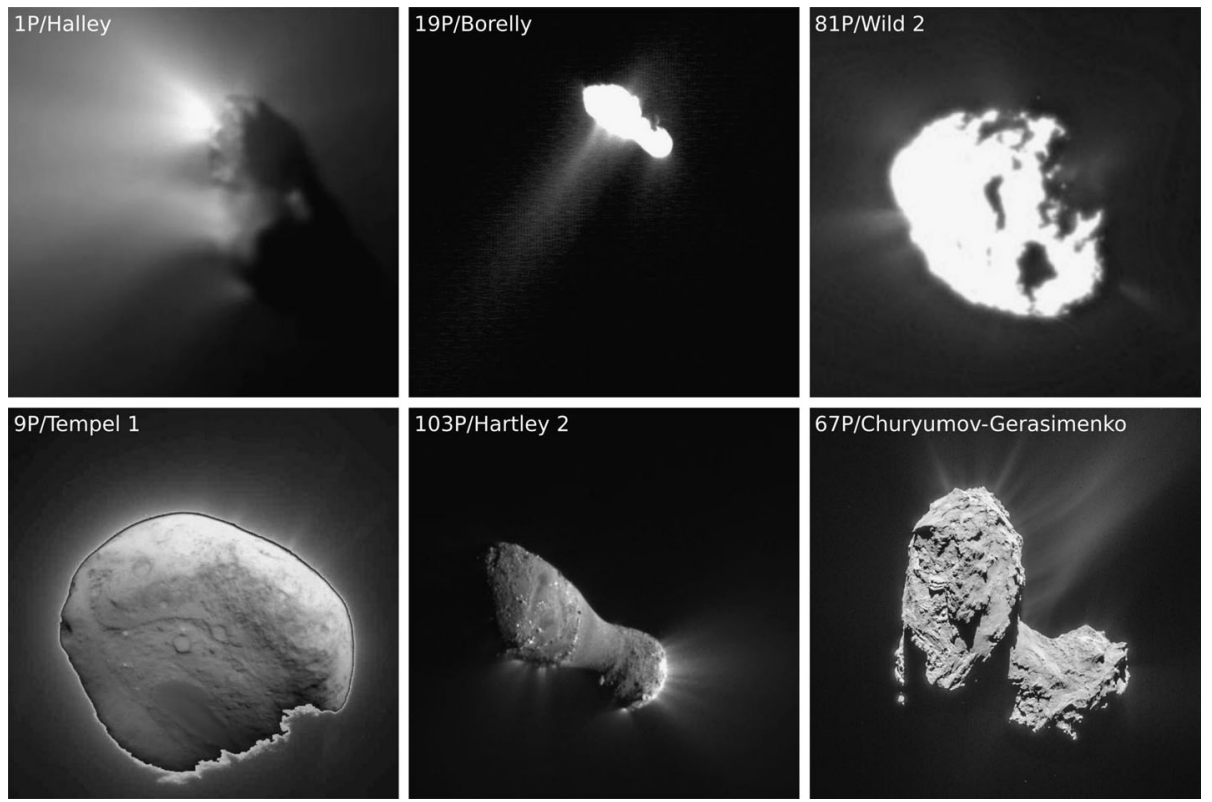

Fig. 1 Cometary activity observed by space missions, in chronological order from left to right, top to bottom

\section{Introduction}

When the Giotto mission flew by comet 1P/Halley in March 1986, it completely changed our understanding of comets and their activity. Among many discoveries, the mission revealed a dark nucleus with little or no exposed ice and a non-isotropic coma. While most of the surface appeared inactive, a few areas gave rise to strong collimated flows of gas and dust, commonly referred to as "jets".

In the following 30 years, five additional cometary nuclei have been imaged by space probes, confirming the picture revealed by Giotto: cometary nuclei are among the darkest objects in our Solar System, and the distribution of gas and dust density above the surface is highly non-isotropic, with ubiquitous collimated features.

While there is no doubt that coma features are collimated streams of dust and gas (for only the density contrast with the ambient coma makes them detectable in images), the cause of this collimation is challenging to establish, and has led to many publications with conflicting definitions for similar observations. One issue, to be discussed in Sect. 2, is that physical processes that are completely unrelated can give rise to the same type of morphological features. It is for instance well established that topographic variations will collimate gas flows and create regions of higher density where the dust can be more easily accelerated. This phenomenon has been detected on various objects. On the other hand, observations also suggest that some localized areas are more volatile-rich than others, with the ice being sometimes directly exposed on the surface. Consistently, this larger amount of volatile material will sustain activity for a longer time that depleted regions, thus leading to stronger gas and dust flows over those areas, and jet-like features. Figure 1 shows how activity looked like for the 6 cometary nuclei images in situ so far. 
Several attempts have been made to classify these features and connect them to processes which can then inform us about the physical and chemical properties of cometary nuclei. A comprehensive review by Belton (2010) proposes the following nomenclature:

- Type I describes dust release dominated by the sublimation of $\mathrm{H}_{2} \mathrm{O}$ through the porous mantle;

- Type II is controlled by the localized and persistent effusion of super-volatiles from the interior;

- Type III is characterized by episodic releases of super-volatiles.

In this description, Type I jet-like features do not appear to be associated to specific morphology and are generally broader and more diffuse than other dust streams. Type II features, also called filaments in the literature display a much more collimated structure and have been traced back to specific regions of cometary nuclei. Finally, Type III are more sporadic events, probably related to micro-outbursts or other explosive processes.

This description was well suited to describe observations acquired from the first flybys (comets 1P, 9P, 19P, and 81P), but does not apply to all features observed by later missions (comets 103P and 67P). In addition, this former nomenclature imposes an interpretation by associating morphological description of jet-like features with some physical processes which are still a matter of debate.

In this paper, we chose to dissociate morphology and physics: we propose a nomenclature that is purely based on observations and define terms that can remain future proof. We will show how our definitions relate to Belton's. In Sect. 5, we will review the potential mechanisms of cometary activity, and discuss whether some processes relate more closely to one specific manifestation of activity or another.

\section{Nomenclature of Cometary Activity}

A spacecraft orbiting a cometary nucleus will typically observe activity: release of volatile and refractory material, as producing an ambient coma and localized regions of enhanced gas/dust density.

\subsection{Ambient Coma}

The ambient coma is also known as "coma background" is present in all observations and may have to be subtracted from the data in order to detect anisotropies. It is a diffuse background of gas and dust, typically enhanced on the day side of the nucleus where sublimation is stronger.

Different volatile species will display different distributions, depending on their sublimation temperature. For instance $\mathrm{H}_{2} \mathrm{O}$ is much more abundant on the day side, while $\mathrm{CO}_{2}$ expands in a more isotropic coma. Of course, this distribution is somewhat affected by local variations of composition on the nucleus' surface, but the diffuse nature of such activity makes it often difficult to associate a specific region with a given gas species abundance. Secondary sources, e.g. ice grains ejected from the nucleus and sublimating at some distance from the surface, may introduce some anisotropy in the distribution. Example of such phenomena can be found in A'Hearn et al. (2011) for comet 103P/Hartley 2 or Bodewits et al. (2016) for comet 67P/Churyumov-Gerasimenko

Likewise, dust also expands in a coma background, accelerated by the gas flows for a few nucleus radii until the gas density becomes too low to provide further acceleration. Beyond 
that limit, dust motion is mostly controlled by solar gravity and radiation pressure, and the expands into general coma, tail and trail.

A more thorough description of the coma background for dust and gas, as well as the prime mechanisms for expansion, and a summary of numerical simulations efforts on this topic can be found in the "Coma" chapter of this book.

\subsection{Collimated Streams}

Collimated streams have been observed around all cometary nuclei, and are always identified as narrow regions of higher density in gas/dust with respect to the ambient coma. At the distances and resolutions considered in the paper (spacecrafts flying by or orbiting cometary nuclei), these features are typically detected by stretching the contrast of images. This is different than for ground based observation which often require special image filtering in order to reveal coma structures (Samarasinha and Larson 2014). The absence of features in our data means that no variations in coma brightness can be detected against the noise in the background, indicating a very low level of activity, or none at all.

While they are easy to detect, naming them and understanding the physics of their formation has been a challenge ever since the first observations (either ground based or in situ). Many authors have described these collimated streams using different names: e.g. "coma structures", "jets", "jet-like features", "plumes", or "filaments". Often the same words are used to describe collimated streams at very different scales, from spacecraft observations (a few tens of $\mathrm{km}$ from the surface) to ground based observations (a few tens of thousands kilometers from the nucleus). In addition, some of these words carry a physical meaning which may not be the correct interpretation of the nature of this phenomenon. A "jet", for instance, implies that the gas/dust stream is being forced out of a small orifice. Although this is a working hypotheses, it has never been observed as such so far. We should also be wary of misinterpretation by people with different knowledge background: "plume" describes accurately a cloud of dust/gas but also carry the general meaning of having different properties (composition, temperature) than its surrounding, and being potentially buoyant. Neither case is necessarily applicable to the case of comets. "Filament" is a better word, and already mentioned in solar physics to describe coronal streamers. It is however seldom used in the cometary literature, where "jet" (although ambiguous) has been the dominating word.

In this paper, we advocate the usage of neutral vocabulary and recommend the usage of "collimated streams" when describing the observations. However, we also recognize that some words are well established in the community and cannot be dismissed totally. We do strongly suggest, though, to restrain from using the single word "jet" and instead prefer the longer version: "jet-like feature". This provides a visual description of the observations that is close enough to what has been already published, while at the same time not claiming definitely that these features are produced by a jetting process.

Note that this definition is based on the morphology of collimated streams themselves and does not assume any relation between a jet-like feature and nucleus surface morphology/composition. This is an important point which will be developed in Sect. 4.

\subsection{Temporality}

All missions prior to Rosetta have been single fly-bys encounters. This means that they provided a snapshot of cometary behaviour at a fixed epoch, with high resolution observations lasting only a couple of hours at best. By orbiting comet 67P for over 2 years, throughout the perihelion passage, Rosetta gave us an unprecedented view into the temporality of activity 
Table 1 Nomenclature of local manifestations of cometary activity

\begin{tabular}{lll}
\hline Description & Classification \\
\hline Long-lasting events, predictable? $\Rightarrow \begin{array}{l}\text { Perennial collimated streams } \\
\text { (jet-like features) }\end{array}$ \\
Short-lasting events, sporadic? $\Rightarrow \quad$ Transient plumes \\
\hline
\end{tabular}

phenomena. In particular, it was possible to observe some jet-like features repeating from one rotation to the next, seemingly arising from the same area, while others were sporadic events appearing only once.

The temporal factor turns out to be extremely important when trying to infer the mechanisms leading to the release of gas and dust, and to the collimates streams we observe. In fact, a single image can often be misleading. Out of the many jet-like features visible at any time, some may last for many hours - rotating with the nucleus, switching off at night and waking up the next morning - while others may only be detected in that single frame. Yet both types of features may appear morphologicaly identical.

Some attempts have been made to include temporality in the nomenclature, e.g. Belton (2010, 2013), Vincent et al. (2016). Unfortunately, they often mix observation and interpretation. This should be taken with caution as establishing the processes behind cometary outbursts remains a challenge. Yet, a clear distinction can be made between different types of activity based on the duration of each event. Our proposed nomenclature is described hereafter and summarized in Table 1.

Cometary missions have observed two categories of jet-like features. While both share the same morphology and can be traced down to specific areas of the nucleus, their dynamics are quite different.

In the most common case, collimated streams appear to last for many hours: they rise as soon as the local temperature enables sublimation, rotate with the nucleus, and wane some time after the local sunset. They will often be reactivated on the next morning, and repeat this cycle for many rotations. For a source more volatile than water, such features has been observed to last for several full rotations, with little or no decrease in intensity during the night (Feaga et al. 2007). We will refer to these features as perennial or long-lasting. Observations suggest that these common streams contain more dust than the surrounding coma (that's why we can detect them) but not necessarily a different speed or composition. A particular case of perennial activity are curtain-shaped streams (i.e. collimated only in one direction) which closely follow the morning terminator and are associated to the sublimation of frost in the early local morning (De Sanctis et al. 2015; Shi et al. 2018). While each source area will only be active for a short time, the active foot print slowly swipes a large fraction of the morning surface and is reactivated every morning, thus we consider them perennial as well.

The second type of jet-like features are transient, or short-lived. They arise suddenly for a few minutes, and may never be detected again from that location. These are traditionally associated to outbursts, i.e. the sudden release of a large quantity of gas and dust, driven by mechanisms that are still debated. While outbursts have usually been interpreted as akin to an explosive decompression, this interpretation is challenged by EPOXI and Rosetta data and the associated models which suggest that the transient release of material can be well explained by avalanches (Steckloff et al. 2016) or topographic collapses (pits, cliffs, Vincent et al. 2016). Spacecraft observations have shown that such events are more likely to occur around perihelion and in the outbound orbit, but not always. They may arise from very different terrains and illumination conditions on the nucleus (Belton 2013; Knollenberg 2015; Vincent et al. 2016; Grün et al. 2016). Some events may also repeat from the same location, 

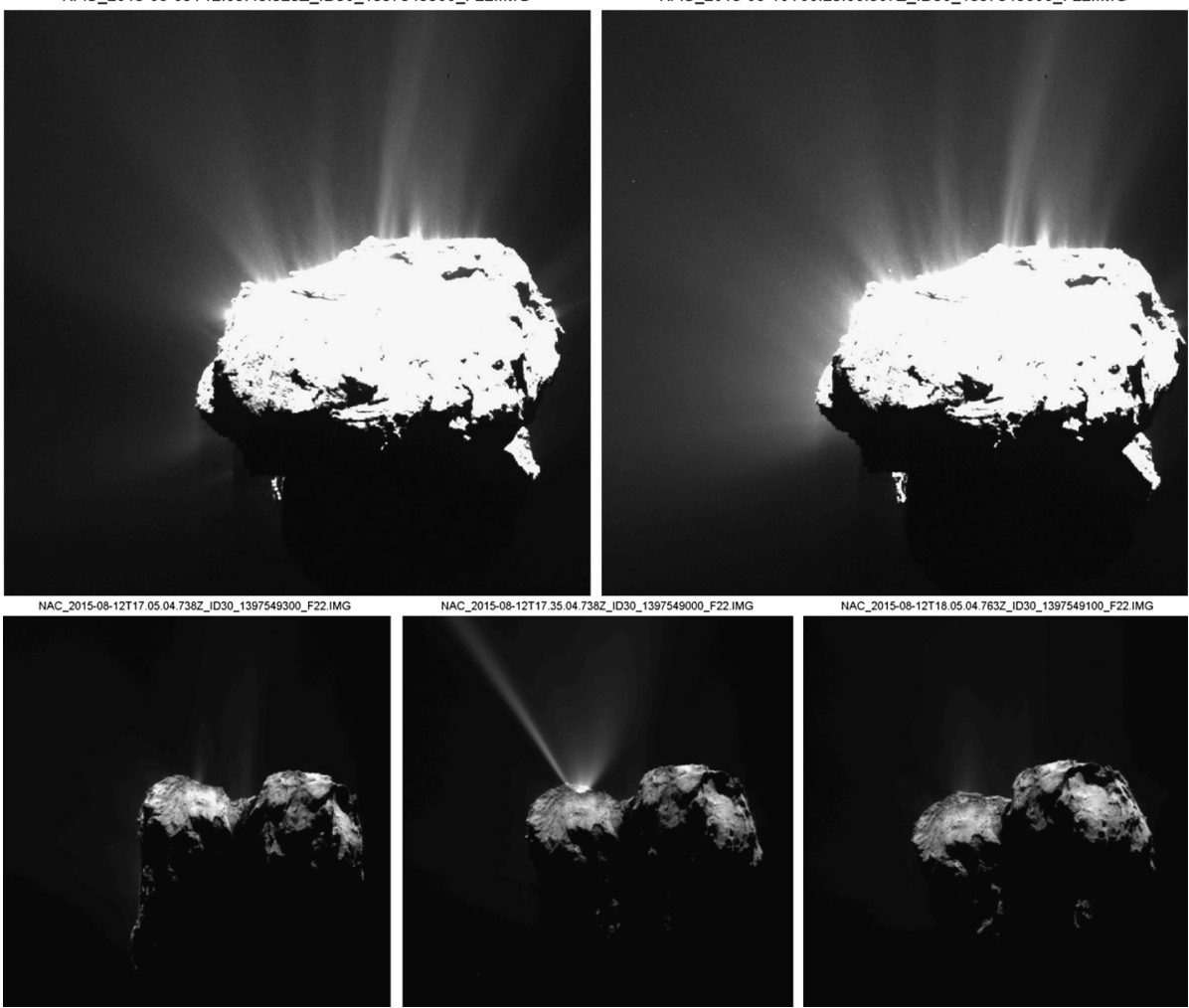

Fig. 2 The two main local manifestations of cometary activity, as observed by the OSIRIS Narrow Angle Camera on board Rosetta, at comet 67P. The top panels show contrast enhanced views of the comet nucleus acquired one rotation apart. One can see the exact same perennial collimated streams consistently repeating from one comet day to the next. The bottom row shows an outburst event, in which a major transient plume appears only in the middle frame, and is not observed $1 / 2 \mathrm{~h}$ earlier or later. Adapted from Vincent et al. (2016)

at different frequencies (from days to months, Agarwal et al. 2016; Oklay 2018). Transient events may lead to different morphologies for the associated dust feature. Vincent et al. (2016) distinguish three categories: Type $A$ are narrow, strongly collimated streams, Type $B$ are broad plumes, and Type $C$ are complex morphologies with several streams of types A and $\mathrm{B}$ at once. The term plume is quite relevant here as the material associated with transient events has often different properties than the ambient coma (an order of magnitude faster, smaller grains, enriched in water ice, see papers cited above).

To summarize, we classify all local manifestations of cometary activity in two major classes: perennial collimated streams and transient plumes, illustrated in Fig. 2. Sub-classes may be introduced when required by the observations, or when the physical process can be clearly established.

Going back to Belton (2010)'s nomenclature, our perennial collimated streams effectively merges the types I \& II, as we seen with Rosetta that we often cannot distinguish between jet-like features related to $\mathrm{H}_{2} \mathrm{O}$ sublimation alone, or other volatiles. Transient plumes are equivalent to Belton's type III, although once again detached from any physical interpretation. 
Table 2 Spacecraft missions to comets

\begin{tabular}{llll}
\hline Mission & Target & Date & $\begin{array}{l}\text { Close approach } \\
\text { distance }\end{array}$ \\
\hline Vega 1 & 1P/Halley & 6 Mar. 1986 & $8889 \mathrm{~km}$ \\
Vega 2 & 1P/Halley & 9 Mar. 1986 & $8030 \mathrm{~km}$ \\
Giotto & 1P/Halley & 14 Mar. 1986 & $596 \mathrm{~km}$ \\
Deep Space 1 & 19P/Borrelly & 22 Sep. 2001 & $2171 \mathrm{~km}$ \\
Stardust & 81P/Wild 2 & 2 Jan. 2004 & $237 \mathrm{~km}$ \\
Deep Impact & 9P/Tempel 1 & 4 Jul. 2005 & $500 \mathrm{~km}$ \\
$\begin{array}{l}\text { Deep Impact } \\
\text { (EPOXI) }\end{array}$ & 103P/Hartley 2 & 4 Nov. 2010 & $694 \mathrm{~km}$ \\
Stardust NExT & 9P/Tempel 1 & 15 Feb. 2011 & $182 \mathrm{~km}$ \\
Rosetta & 67P/C-G & 2014-2016 & Orbital \\
\hline
\end{tabular}

\section{History of Observations}

\subsection{General Introduction}

Consideration of studies of cometary activity in a historical context shows a progression in which improvements in technology and data acquisition have built upon previous knowledge to further our understanding of the physical processes at work on cometary nuclei. It has been known from many decades of ground-based observations that active areas on the nucleus can produce features in the coma. Linear filaments, corkscrews, arcs, and spirals are detected in images of both dust and gases, with the different morphologies reflecting the comet's activity, the dynamics of the nucleus and the viewing geometry. These features are often used to infer the nucleus' physical properties and to constrain characteristics of the comet's emission (e.g., features extending thousands of kilometers from the nucleus indicate that the material must be highly collimated). Unfortunately, the large scales of ground-based observations shed little light on the interaction between the nucleus and coma or about the mechanisms producing the observed features. With the advent of spacecraft missions that could obtain images of the resolved nucleus, it became possible to start investigating the nature of the activity itself. Although most missions were simple flybys that captured only "snapshots" of the nucleus' behavior, each successive visit revealed additional details about the physical characteristics of coma structures. From the very first images of 1P/Halley, through the long-term observations of 67P/Churyumov-Gerasimenko, individual comets show different types and scales of activity changing on a variety of timescales, and the aggregate of these measurements has provided the necessary background to better understand the mechanisms that drive cometary activity. We outline here a brief history of the different comet missions, with a summary of some of the highlights of each. A timeline of all missions is given in Table 2.

\subsection{P/Halley Flybys (Vega $1 \& 2$ and Giotto Spacecraft)}

1P/Halley, the only cometary mission target to date that is not a Jupiter Family Comet, was the first comet to have its nucleus resolved in spacecraft images (Sagdeev et al. 1987). An important conclusion from these observations was the confirmation that the nucleus did indeed exhibit isolated active areas on its surface that produce features in the coma (Keller et al. 1987; Larson et al. 1987). Unlike objects visited later, however, Halley's inner 
coma was so dense that it masked the details of the nucleus' surface. This complicated any detailed analyses involving the interaction between the surface and coma and the processes that generated the activity. A number of attempts were made to use the spacecraft images to extract information about the locations and other properties of the active areas on the surface (e.g., Celnik and Schmidt-Kaler 1987), but ambiguities in the spacecraft viewing geometry, nucleus shape and complex rotation state (e.g., Belton et al. 1991; Stooke and Abergel 1991) made it difficult to study the coma-nucleus interactions. There is a growing interest in revisiting the Halley spacecraft observations, using modern image processing and analysis techniques, to maximize the return from these missions and provide more up-todate comparisons to the more recently studied comets.

\subsection{P/Borrelly (DS1)}

Because DS1 was a technology demonstration flight redirected to comet Borrelly after the end of its primary mission, it obtained only a few high resolution images near close approach. However, this limited dataset represented the best images of a comet's nucleus available at the time and showing a variety of different coma features, including a highlycollimated linear stream, a broad fan, and a small rapidly changing loop feature (Soderblom et al. 2002). Although the collimated streams exhibited little detail, they provided important insight that foreshadowed what was to come in future missions.

During its approach, DS1 tracked the strong, highly collimated jet-like feature for some time. This stream was unambiguously connected to a long-term coma structure that had been detected in ground-based observations for decades, and whose constant orientation in inertial space indicates that it is aligned with the nucleus' spin axis (Farnham and Cochran 2002). DS1 close approach data showed that this feature arises from a central basin at the waist of the elongated nucleus, but the limited spatial resolution of the comet's surface make it impossible to identify the source with any specific geologic structures on the surface. Even so, this was the first measurement of a common feature that can be traced from its origin on the nucleus to $>10000 \mathrm{~km}$ from the nucleus, and it confirmed that features seen in groundbased observations do indeed reflect the comet's surface activity (though the comet's rotation and observing geometry can obscure the connections).

DS1 also detected the first rapid changes in a comet's innermost coma, recording a "loop" structure at the terminator where dust produced from an unilluminated portion of the surface is emerging into sunlight (Boice et al. 2002). The appearance of this structure changed on timescales of less than a minute, and it was believed that the rapid variability is related to the shutdown in activity after the source had recently entered darkness. Again, the restricted dataset limits any detailed analysis of this phenomenon, but it represents the first occasion of features changing on very short timescales.

\subsection{P/Wild 2 (Stardust)}

The coma of Comet Wild 2 around perihelion is dominated by a persistent, large-scale sunward fan that is centered on the nucleus' spin axis (Schulz et al. 2003; Farnham and Schleicher 2005). Unfortunately, the Stardust flyby occurred late in the apparition (98 days after perihelion), after this feature had disappeared due to the changing seasonal variations. Thus, not only was the primary fan absent during the encounter, but the surface of the nucleus where it originated was unilluminated and could not be studied. Stardust did record a number of small, isolated jets around close approach, however, and Sekanina et al. (2004) 
performed an analysis of these structures. Their results showed the features were widely distributed around the surface, with no obvious correlations in their source regions. It should be noted that this work only projected the jet-like features back to their intersection with the surface of the triaxial ellipsoid that was fit to the nucleus, and a more complete analysis that investigates the source regions with respect to the actual shape model of the nucleus might reveal more meaningful information about specific correlations to the topography at the origins of the activity.

Sekanina et al. (2004) also found that several collimated streams arose from the night side of the nucleus, confirming that activity can persist for some time after the site is no longer illuminated. Given the locations of these features and Wild 2's 13.5-hr rotation period (Farnham et al. 2010) these sources may have remained active for hours after local sunset. Unlike in comet Borrelly, the jet-like features displayed no temporal variations that would suggest the activity might be shutting down.

\subsection{P/Tempel 1 (Deep Impact)}

Comet 9P/Tempel 1 is unique in two respects: First, it was the target of a large-scale impact experiment designed to investigate the nucleus' sub-surface characteristics. This experiment offered the unprecedented opportunity to explore the conditions that contribute to cometary activity. Second, it was a spacecraft target on two consecutive apparitions, offering the first opportunity to detect changes in the nucleus produced by the comet's long-term activity.

As with comet Wild 2, Tempel 1 is known to exhibit a persistent sunward fan in its coma during the pre-perihelion portion of its orbit. Although continuum images of this feature show no short-term changes (e.g., Lara et al. 2006), CN observations resolved it into a corkscrew that oscillated on timescales consistent with the comet's 41-hr rotation period (Farnham et al. 2005; Schleicher 2006; Boehnhardt et al. 2007). Both observations indicate that the feature was produced by an active area located near the nucleus' south pole. This primary fan had faded in ground-based observations by the time of the Deep Impact (DI) flyby (1 day before perihelion), the spacecraft recorded a diffuse jet-like feature arising from the south pole that likely represents residual activity from the primary fan. This stream is too diffuse to be visible against the illuminated portions of the nucleus, limiting any direct connection to surface features that might indicate its origin. However, projecting the linear structure seen beyond the limb back toward the surface suggests it is associated with the large smooth flow that encompasses the nucleus' south pole (Farnham et al. 2007; Vincent et al. 2010).

The connection between the activity and the smooth terrain was confirmed by other features seen in the DI images. Additional polar activity was observed on the nightside beyond the terminator, where dust is seen in a string of connected sources against the dark surface. Fortuitously, after the impact experiment, this region became illuminated by sunlight reflected off the rising ejecta cloud, and the indirect lighting revealed another, previously undetected, smooth patch on the surface, with the string of dust features aligned along its edge (Farnham et al. 2007; Thomas et al. 2007). This was the first direct observation of cometary activity arising from a scarp or vertical surface, a phenomenon that has been observed on many occasions since. The relationship between active areas and vertical faces provides a natural explanation for the persistence of cometary activity, in that a scarp can erode backward, continuously revealing fresh volatiles, while the vertical surface prevents insulating dust from accumulating and choking off the activity.

The Deep Impact IR Spectrometer obtained the first high quality measurements of volatiles in the coma, hinting at the processes that drive a comet's activity (Feaga et al. 
2007). Notably, the southern polar region of Tempel 1 is dominated by $\mathrm{CO}_{2}$ emission, while $\mathrm{H}_{2} \mathrm{O}$ is most prominent near the sub-solar point. From these observations, it is clear that the gas production is heterogeneous, with $\mathrm{CO}_{2}$ concentrated in the region where the highest activity levels are seen. This suggests that the distribution of highly volatile material could play as significant a role in the activity as the illumination conditions. Furthermore, the presence of $\mathrm{CO}_{2}$ near the south pole suggests that it is the driver of the activity seen on the nightside of Tempel 1 (and by extension, on Wild 2 and other comets). Because $\mathrm{CO}_{2}$ is more volatile than $\mathrm{H}_{2} \mathrm{O}$, even low levels of lingering heat can apparently continue to generate activity long after the region is in darkness.

DI also detected ice on the surface of Tempel 1, in the form of several small patches on the "flat top" that had just rotated into sunlight (Sunshine et al. 2006). These patches were associated with small, narrow streams whose proximity indicates they are related to the ice, but whose morphology suggests that they do not actually arise from it. Instead, the jet-like feature origins were found to coincide with small dark features adjacent to the ice fields, and it was interpreted that those jets were the result of volatile sublimation which dragged sub-surface ice and dust into the coma (Farnham et al. 2007). Some of this material then falls back to the surface in the vicinity of the jets, producing the ice patches that were observed. It is notable that an increased concentration of $\mathrm{CO}_{2}$ was detected in the coma around these jets, possibly permitting nighttime activity that would result in the ice fields (which are likely short-lived) being seen shortly after local sunrise.

Another constraint on the mechanisms that contribute to cometary activity comes from the DI impact experiment. Using IR sequences of the ejecta and modeling of the excavation event, Sunshine et al. (2007) showed that water ice exists within a meter of the surface, even at a site that revealed little evidence for activity in its pre-impact state. This suggests that a thermal wave doesn't need to penetrate very deeply to sublimate volatiles. Furthermore, on vertical surfaces that don't build up an insulating layer, ices are likely to be much closer to the surface, enabling the formation of isolated gas and dust streams.

In 2011, comet Tempel 1 was visited by the Stardust spacecraft on its extended mission (Stardust NExT; SDN), providing the first comparisons of a comet on two subsequent apparitions. Combining the observations of the regions observed by both DI and SDN provides a means of investigating the surface evolution over the course of slightly more than one orbit. Thomas et al. (2013) discuss these changes, the most prominent of which is the recession, by more than $50 \mathrm{~m}$ in places, of the leading edge of the south polar smooth flow. The loss of as much as $108 \mathrm{~kg}$ of material from this region confirms that the scarp is a significant contributor to the activity arising from the polar region.

As far as activity, the SDN flyby occurred later in the apparition ( +33 day), so the nucleus' production rates were much lower than during the DI flyby. Even so, it is notable that no activity was detected from the sources seen in the DI observations, even though the viewing geometry was such that the coma features should have been seen if they were present. The "flat top" region of the surface containing the ice patches was on the limb during the SDN departure, but it was also in darkness, so it is not known whether the small jets were still active at this point in the orbit. The conspicuous differences in this respect sets a tight constraint on how the diurnal and seasonal activity evolves throughout the orbit.

SDN did observe a number of isolated active areas (Farnham et al. 2013), some of which arise from unilluminated regions, so it is not possible to evaluate their origins. The most striking features, however, are a cluster of narrow jets seen on the limb near close approach. These are traceable back to their sources on the surface, and Farnham et al. (2013) showed that they all arise from a terraced structure that forms the boundary between a rough highland area and a smooth lowland region. This further confirms that jet activity is frequently associated with vertical topography. 


\subsection{P/Hartley 2 (Deep Impact Extended Investigation)}

Little was known about comet Hartley 2 before the encounter, other than it is a member of the rare family of hyperactive comets (those that emit more water for their size than would be expected from water production models). As with comet Tempel 1, the IR spectrometer observations show $\mathrm{H}_{2} \mathrm{O}$ and $\mathrm{CO}_{2}$ emissions in the inner coma, with links to the continuum features seen in images at visible wavelengths (Protopapa et al. 2014). Unlike Tempel 1, however, the continuum seems to be exclusively associated with the $\mathrm{CO}_{2}$ in the coma, with an almost anti-correlation to the $\mathrm{H}_{2} \mathrm{O}$. This strongly indicates that hypervolatiles are driving the jet activity in Hartley 2. There is also a thin layer of water ice seen on the morning terminator, visible in the approach images, that might indicate that water is recondensing or ice grains are falling back to the surface during the night (Sunshine et al. 2011). The rest of the surface appears to be free of ice, suggesting that the recondensed material rapidly sublimes away when in sunlight.

Hartley 2 exhibits numerous isolated active regions, with a strong concentration on the end of the smaller of the two lobes of the nucleus. A second concentration is located along the evening terminator on the side of the larger lobe, and a number of more isolated jet-like features are distributed around the rest of the nucleus, including some that arise from the night side (Farnham et al. 2011). The observed streams range from narrow, highly collimated structures to broader, diffuse fans. Many of the jets can be traced back to their origins at scarps (including the one at the edge of the nucleus' smooth neck region), rimless depressions, and dark features that may be holes or vents (Bruck Syal et al. 2013). Modeling of the activity during approach and departure indicates that the active areas seen at close approach are the main ones on the nucleus. The activity from these sources increases and decreases with rotation/insolation, but doesn't tend to turn off completely. Furthermore, there is little evidence that there are large sources that are inactive at the time of close approach but turn on under different illumination conditions (Farnham 2012).

In addition to the ice seen on the surface, the DI IR spectrometer also identified water ice in the coma of Hartley 2 (Protopapa et al. 2014). Much of this ice is in the form of micronsized particles located in the jets emanating from the small-lobe. Their correlation with the $\mathrm{CO}_{2}$ emission indicates that the icy grains are being dragged from the nucleus' subsurface by the $\mathrm{CO}_{2}$. The grains sublime in the coma, producing a secondary, extended source of water that explains the comet's perceived hyperactivity.

A second population of grains, never seen in previous comet encounters, was also discovered around the nucleus of Hartley 2. These large, individual entities are 10s of centimeters in size, and likely consist of fluffy aggregates of mixed dust and icy particles (Kelley et al. 2013). Although Hermalyn et al. (2013) mapped the positions and motions of a selection of these grains, their dynamics are not well understood. Some show no motion, and will likely return to the nucleus, others are exceeding the escape velocity but on paths that are not radial, while others are clearly escaping from the nucleus. Because of the oddities of the particles' motions, there is no information about the origin of these grains, or whether the aggregates came directly from the nucleus or formed in the coma.

A final phenomenon of interest observed by the DI IR spectrometer was a water feature arising from the smooth neck region. What makes this feature unusual is that it has no continuum associated with it. The smooth neck has been interpreted as a gravitational low, where dust fallback has filled in the depressions, which may be producing an insulating layer that chokes off emission from the underlying surface. Presumably, part of the fallback includes icy grains and these are likely subliming away from the surface to produce the water feature above the neck. The fact that the escaping water does not entrain any dust grains 
should provide information about the mechanisms by which dust features are produced (e.g., gas arising directly from the surface may not provide a means of lifting or accelerating the dust grains).

\subsection{P/Churyumov-Gerasimenko}

Comet 67P/Churyumov-Gerasimenko has been observed by Rosetta from end of March 2014 to end of September 2016, from 4.3 AU inbound to 3.8 AU outbound. In this period, the distance spacecraft-nucleus varied from $5.10^{6} \mathrm{~km}$ down to the surface. As this paper deals with local manifestations of activity, we will focus on the epoch August 2014-September 2016, during which the spacecraft orbited mostly between $10 \mathrm{~km}$ and $300 \mathrm{~km}$ from the nucleus (with the exception of two far away excursions in the sunward and anti-sunward directions).

Activity is a continuous process on comets, and its intensity is strongly driven by the amount of input energy (heliocentric distance). From Rosetta observations, we can roughly define four main epochs showing significant variations in activity pattern: wake-up, equinox and perihelion, post-perihelion.

\subsubsection{Wake-Up}

The beginning of the wake-up phase is not known, as the sensitivity of distant observations is limited. OSIRIS/Rosetta observations show that the nucleus was already faintly active in our first observations (4.3 AU), displaying a brightness profile slightly broader than star-like, a signature of the developing coma (Tubiana et al. 2015). We do not know if this comet is active at larger heliocentric distance, but it can be investigated by a sample return mission such as CAESAR, which will approach the comet at its aphelion.

From onset of activity to arrival at the comet in August 2014, Rosetta observed the gradual development of the ambient coma, and started to resolve collimated streams at various scales. Among these features we detected several streams which appear to originate from the bright, ice-rich concavity in between the two lobes of the nucleus. These jet-like features are interpreted as the combination of enhanced volatile content in this specific area and a focusing effect by the topography (Lara et al. 2015; Lin et al. 2015).

It is important to note that the wake-up of activity was not only a slow and quiet process. Tubiana et al. (2015) reported an outburst at the end of April 2014, which increased the brightness by $65 \%$. Unfortunately, the nucleus was unresolved in OSIRIS data at that time, and we do not know how the surface was modified by this event.

After Rosetta arrived at 67P on 06 August 2014, the spacecraft remained about 6 months at distances lesser than $100 \mathrm{~km}$ from the nucleus. Thus Rosetta was deeply into the coma, in a prime location to observe the developing activity close to the surface. Vincent et al. (2015a) report on this evolution from arrival (August 2014, $3.6 \mathrm{AU}$ ) until equinox (May $2015,1.6 \mathrm{AU})$. They observed that the footprints of jet-like features appear to follow the subsolar latitude, and originate from an ever larger area of the nucleus as the heliocentric distance decreases, in agreement with prediction from thermal models such as Keller et al. (2015). They also note that activity seems to switch on and off with the Sun rising and setting over a given region. This suggests that such activity is driven by the diurnal heat wave sublimating water ice, and must originate from the surface or the very shallow subsurface.

Three-dimensional inversion of jet-like features to find their footprints show a correlation with topographically rougher areas, most notably taluses of receding cliffs and large pits (Vincent et al. 2015b,a), as it had been observed previously on other comets (e.g. 
9P/Tempel 1, Farnham et al. 2013). One should note, however, that the link between morphology and jet-like features is very complex. As described by Crifo et al. (2005) and many later authors, collimated streams of gas and dust may seem to arise from well defined location which are not intrinsically different from any other place. The collimation is mostly due to interactions of the flow with the topography, which explains why one is more likely to observe jets from cliff and pits rather than smooth, flat terrains. A more recent example including numerical simulations of this effect can be found in Shi et al. (2018).

It is nonetheless important to stress that the footprints of most jet-like features observed on 67P can be related to the remains of collapsed cliffs, where regressive erosion exposes icy layer previously insulated by a layer of dessicated material (Vincent et al. 2015a; Oklay et al. 2016). It seems that both topography and availability of volatile material play an important role in forming the observed features.

\subsubsection{Equinox and Perihelion}

The equinox crossing in May 2015 triggered a increase in cometary activity which cannot be solely explained by the reduced heliocentric distance. Due to the peculiar shape of the nucleus, and the high obliquity $\left(52^{\circ}\right), 67 \mathrm{P}$ experiences strong seasonal effects. One important consequence is that the southern hemisphere remains in quasi polar night $80 \%$ of the orbit, being illuminated only for about 1 year from one equinox to the next (Keller et al. 2015). This means that a large amount of ice can build up and be preserved on this dark side until the inbound equinox, when it is suddenly illuminated for the first time in several years (Choukroun et al. 2015). This translates into an increase in the production rate (Hansen et al. 2016) and in the number of jet-like features observed from Spring 2015 onward (Lai et al. 2016).

In addition to the increase in number of observed jet-like features, we also note that activity start to persist for some time beyond the evening terminator. Shi et al. (2016) have shown that this time lag can be used to derive a thickness of the dust layer covering the buried volatile material. It typically corresponds to a few $\mathrm{mm}$.

This epoch is also when Rosetta started to observe significant changes on the surface (transient pitted patterns, regressive erosion, boulder movement, fracture extension, see ElMaarry et al. 2017).

This increase of activity can also be traced by the numerous transient event detected after July 2015 onward. Despite a quasi constant monitoring, no outbursts were detected after the distant one caught in April 2014 (see previous subsection). Vincent et al. (2016) show that many major events were observed (roughly one every other comet rotation), each releasing several tens of tons of material in a few minutes. We found that outbursts occur about every 2.4 nucleus rotations. They are comparable to what had been observed on previous comets. Like for perennial collimated streams, transient plume also appear to originate from areas close to the subsolar latitude, although not always. The spatial distribution of their footprints on the nucleus correlates well with morphological region boundaries, especially areas marked by steep scarps or cliffs. In some cases, the plumes could be linked to the sudden collapse of a cliff, but many other events remained to be explained.

\subsubsection{Post-perihelion}

Further work on outbursts (e.g. Lin et al. 2016; Agarwal et al. 2016; Oklay 2018) show that their frequency decreased after perihelion, but did not stop and transient events could be observed until the end of the mission, more than one year after perihelion. In fact, this type 
of activity turns out to be extremely asymmetric: Rosetta recorded one single event in the inbound phase down to $1.3 \mathrm{AU}$, and several hundred from one month before perihelion to the end of the mission (outbound arc, 3.8 AU). This hints at outbursts being driven not by daily processes, but rather by the seasonal heat wave reaching deeper regions of the nucleus, and perhaps more volatile ices.

This asymmetry can also be found in the overall production rate (Hansen et al. 2016) and the number of jets seen by the cameras on board Rosetta (Lai et al. 2016; Kramer et al. 2017). It is compatible with our understanding of the thermal processes involved (Keller et al. 2015).

\section{Active Sources and Morphological Changes}

\subsection{Active Sources}

If the coma present anisotropies, and activity manifests itself as collimated streams of gas and dust, it is logical to assume an anisotropic distribution of sources on the nucleus. That is, areas where conditions are more favourable to the release of material, e.g. a local increase in volatile material, fine dust more easily liftable, or concavities leading to a heat trap. But such active regions are more elusive than it seems, and have been debated ever since the first Giotto images of comet 1P/Halley in 1984. Indeed, this data revealed a relatively homogeneous nucleus surface with almost no ice, although many collimated streams were detected in the coma (Keller et al. 1987). This led the community wonder whether we actually need active and inactive areas to account for such activity (see Crifo and Rodionov 1997 and Crifo et al. 2002 for an exhaustive discussion). The current consensus is that active regions do exist but the topography is a major player in focusing the gas/dust flows.

Analytical examples and numerical simulations show that it is possible to create a jetlike feature from a flat surface if the ice distribution is not homogeneous (Knollenberg et al. 1996), but observations show that this situation is seldom encountered on cometary nuclei. To understand the link between activity and surface morphology, we typically measure the position of the same jet-like features across several images, and use this information to perform a tri-dimensional stereo reconstruction of the stream geometry. Then one can calculate the intersection between the jet and the surface, keeping in mind that jets give only a faint signal and are mostly seen in limb pointing observations which may hide first few meters (or tens of meters) of the stream if the source lies beyond the local horizon. Inversion techniques like these, from spacecraft observations at close distance, are described in details in Farnham et al. (2007, 2013), Vincent et al. (2015a), Shi et al. (2016) and references therein. We find that jets are more typically linked to rough terrains, where the topography present many small cavities that are very efficient at collimated surrounding streams of gas.

On comet 67P, the rendez-vous nature of the Rosetta mission allowed the teams to investigate further the potential source locations inferred from 3d-inversion of jets. We found that many features appear to arise from cliffs, with the topography naturally collimating flows of gas and dust created in the vicinity (Vincent et al. 2015a). It is also important to note that the cliffs almost always display a talus enriched in water ice, thus providing a volatile input, and also indicating a recent collapse - signature of ongoing erosion (Oklay et al. 2016; Fornasier et al. 2017). Transient events associated to such collapse have also been observed on 67P (Vincent et al. 2016; Grün et al. 2016; Pajola et al. 2017). Other comets show similar link between activity and morphology (Farnham et al. 2007, 2013), and also with landslides in areas covered with fine dust like on comet 103P (Steckloff et al. 2016). 
We also have evidence for jet-like features arising from smoother terrains Shi et al. (2016, 2018) although modeling shows that one still need some topographic variation to support the collimation (Shi et al. 2018).

Overall, it seems like perennial streams preferentially arise from areas where the topography can collimate the flows of gas and dust, with a preference for places where there is more volatile material available. We note that this does preclude activity to arise elsewhere, only that it is clearly where jet-like features are the most visible.

Forward modeling of cometary activity, and link to topography, and particularly the interaction of dust and gas flows in "jets", have been published by many authors. Prominent results can be found for instance in Crifo et al. (2005), Kramer and Noack (2016), Marschall et al. (2017), Shi et al. (2018). Such simulations provide a strong support for the topography driven model with a very repetitive coma structure with each nucleus rotation, with $90 \%$ correlation between simulation and measured intensity around the nucleus (Kramer and Noack 2016).

Still, topography does not explain everything. The transient nature of outbursts suggest that some areas are likely to contain reservoirs of volatile material, released in a sporadic events when the right conditions are met. This phenomenon has been well characterized by the gas related instruments on board Rosetta. For instance, the ROSINA (Rosetta Spectrometer for Ion and Neutral Analysis) analysis has revealed that there is a strong correlation [29 out of 36] between $\mathrm{CO}_{2} / \mathrm{H}_{2} \mathrm{O}$ ice patches and outburst locations (Kramer et al. 2017): $\mathrm{CO}_{2}$ spots are likely candidates for later outbursts and remain active many months after the first events have been observed, indicating the presence of a reservoir extending into the subsurface. Similarly, Oklay (2018) have reported more than 20 outbursts originating from the same location in the Imhotep region of $67 \mathrm{P}$ over a period of 1 year, in the outbound arc of 67P's orbit. This once again suggests that flows from this area relate to a large buried reservoir of ices.

\subsection{Morphological Changes}

If topography affects activity, one may also ask about the reciprocal. As cometary material is ejected away from the nucleus, this must change the surface.

First evidence for topographic changes in relation to activity were reported by Thomas et al. (2013) for comet 9P. After one orbit, they observed the retreat of a scarp with downward erosion of $>20 \mathrm{~m}$, and morphological changes of depressions in smooth terrains. Similar large scale changes were observed on comet 67P (Groussin et al. 2015). In addition, Rosetta also discovered many other types of changes, like fault extension, cliff collapse, transport of large boulders, summarized in El-Maarry et al. (2017) and Chapter "Morphology" of this book.

Dust transport from activity (Thomas et al. 2015; Lai et al. 2016) also play a major role in blanketing and unveiling the topography.

In addition to these large scale changes, many small scale $(<10 \mathrm{~m})$ surface modifications have been detected and can often be associated to the sudden release of material, for instance forming small pits (Agarwal et al. 2016; Vincent 2018; Oklay 2018). A prominent example from comet 67P is shown in Fig. 3.

Overall, it is clear that activity affects the evolution of the surface. Its long term effects on the global shape of the nucleus starts to be understood (see for instance Vincent et al. 2017) but most of the small scale effects have only been revealed by Rosetta and remain to be explained. 

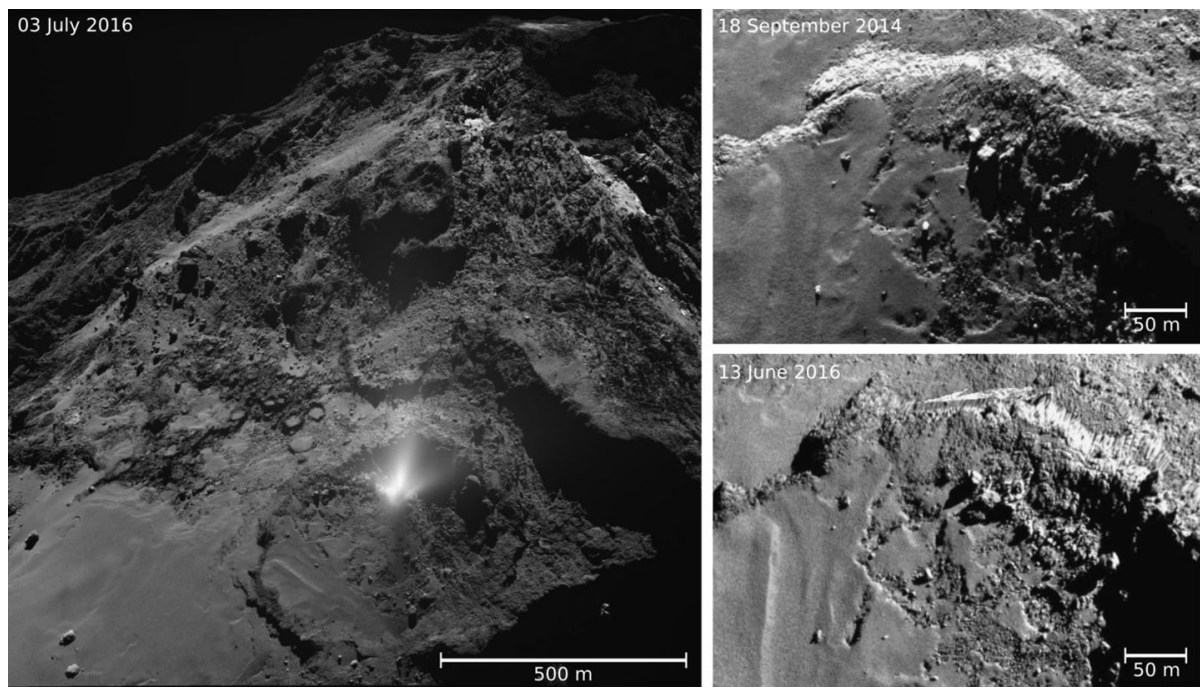

Fig. 3 Left: Rosetta/OSIRIS Wide Angle Camera image of the outburst plume obtained on 03 July 2016. Right: zoom in the same region before and after perihelion. Although the 03 July 2016 outburst is the only one observed at close distance, Rosetta has detected more than 20 events from this same area during the two years of the mission. The surface has changed significantly and numerous pits have been formed Oklay (2018), Vincent (2018)

\section{Nature of Activity}

A basic goal of the Rosetta mission was to learn more about the nature of cometary activity during the long observation time. Cometary activity is the escape of gas molecules by sublimation of ice and of dust grains from sub- $\mu \mathrm{m}$ to $\mathrm{dm}$-size from the nucleus. It is widely assumed that the dust is accelerated by the gas drag (Finson and Probstein 1968); alternative explanations consider electrostatic forces (Horányi et al. 2015). While the dust in perennial jets seems to depart from the surface with almost zero velocity, material ejected in transient events escapes much faster and the trajectory may be controlled by the initial impulse.

The gas pressure provided by sublimating water molecules at perihelion temperatures is in the order of $1 \mathrm{~Pa}$ (Skorov et al. 2017). This is enough to accelerate grains up to a few decimeters in size against the weak gravity but may not be sufficient to overcome the cohesion of cometary matter and actually detach the grains of the surface. This cohesion can be due to van der Waals attraction (Chokshi et al. 1993) and stronger forces like sintering processes (Thomas et al. 1994; Kossacki et al. 2015) or chemical interactions.

According to measurements (Attree et al. 2018a; Thomas et al. 2015; Mannel et al. 2016), the tensile strength of cometary matter reaches several Pa to $\mathrm{kPa}$ depending on the scale of observations. In such a scenario of strength higher than the gas pressure one has to explain how dust can be lifted off and how activity is maintained since an isolating dust crust may be formed. As a consequence the comet should become inactive after short time (Kuehrt and Keller 1994). This phenomenon of self-extinction of activity was observed in comet simulation experiments (Grün et al. 1993) but obviously not at comets. This conundrum is sometimes referred to as the activity paradox (Blum et al. 2014).

There are several ways out of this paradox that will be discussed in the following. When the dust to ice volume ratio is $\ll 1$ dust grains do not touch each other and cannot form a cohesive lattice structure. They escape naturally with the subliming gas. However, according 
to observations (Rotundi et al. 2015) this is an unlikely scenario since dust seems to be the major component in comets. An enhanced gas pressure to crack the cohesive forces would also help. It could be achieved by considering ices with a low sublimation enthalpy. However, such supervolatiles like $\mathrm{CO}_{2}$ and $\mathrm{CO}$ do not dominate the volatile composition (Hässig et al. 2015) and are probably present only in deeper layers where the heat flux from Sun is weak. Additional heat sources in the nucleus, e.g. by chemical reactions (Miles 2016) or phase transition from amorphous to crystalline water ice (Marboeuf et al. 2012) can also raise the gas pressure but there are no direct observations that prove the existence of such exothermic processes. A high pressure can also be achieved according to the pressure cooker effect (Kuehrt and Keller 1994). This needs basically closed pores with a good thermal contact to the surface but is difficult to believe in material with $70 \%$ porosity (Pätzold et al. 2016). Finally, Vincent et al. (2012, 2015a), Skorov et al. (2017) proposed that fractures, ubiquitous in cometary material, may act as nozzles through which the gas is accelerated. This would increase the gas velocity, thus the drag force applied to the dust.

An other way out of the paradox is a local weakening of the cohesive forces that can be broken by the gas flux. Thermal fatigue is too slow (Delbo et al. 2014; Attree et al. 2018b) to have such an effect but locally weak binding forces can be reached by other effects that were not always considered in models or lab experiments.

When assuming that cohesion is mainly caused by van der Waals forces (Chokshi et al. 1993; Kuehrt and Keller 1994; Güttler et al. 2009; Skorov and Blum 2012) between spherical grains with radii R1 and R2 one can derive the contact force (Chokshi et al. 1993) as

$$
F_{c}=3 \pi R \gamma
$$

where $\gamma$ is the surface energy per unit area and $R$ is the reduced radius

$$
R=\frac{R_{1} R_{2}}{R_{1}+R_{2}}
$$

According to the model of Johnson et al. (1971) the cohesive strength $Y$ of a monodisperse medium consisting of spheres of radius $R$ is given by:

$$
Y=\frac{3(1-p) N_{c} \gamma}{4 R}
$$

where $p$ is the porosity, $N_{c}$ is the average number of contact points per particle. Interestingly, this strength does not depend on elastic parameters of the grains.

In the following the importance of several parameters that describe cometary surface layers is discussed to figure out how dust clusters of low of cohesiveness can be formed and, finally, be eroded by the gas.

\subsection{Material Properties}

The stickiness, given by the surface energy per unit area (see table in Chokshi et al. 1993) can vary by orders of magnitude. Water ice is a good glue $\left(\gamma=0.37 \mathrm{~J} \mathrm{~m}^{-2}\right)$, quartz is not $(\gamma=$ $0.025 \mathrm{~J} \mathrm{~m}^{-2}$ ). Therefore, the composition of the surface layer is of importance. However, at low temperatures, typical for cometary ices, the stickiness of ice and dust are more similar than at higher ones as they occur on Earth (Gundlach et al. 2015). 


\subsection{Dust/Ice Ratio}

The dust/ice mass ratio $\chi_{m}$ derived for comet $67 \mathrm{P}$ is not completely understood but Rotundi et al. (2015) derived a value in the range 2 to 6 . The dust/ice volume ratio $\chi_{v}$, that is more important for the geometric structure (and thus for $N_{c}$ ) if we assume that small and large dust grains have the same porosity, results by multiplying $\chi_{m}$ with the density ratio of ice and dust. It is, therefore, considerably smaller (by about a factor 3) than $\chi_{m}$. A $\chi_{v}$ that is not too large (in the order of one or lower) gives a small scale mixture of dust and ice grains where the dust doesn't get the chance to intensely interlink over large distances and to form stable clusters to some depth. Thus the surface can be easily cleared from dust in the sublimation process independently on the cohesion. At the surface this ratio may be enhanced compared to the bulk by ice depletion due to sublimation process.

Deep Impact and Rosetta measurements (Sunshine et al. 2006; Capaccioni et al. 2015) conclude that there are only small amounts of ice on the surface of comets. However, lab results (Yoldi et al. 2015) demonstrate that ice can be masked in IR spectra. Up to now, it is not completely understood to what extend and to what depth the superficial dust/ice ratio is higher than that of the bulk. The problem of determining the ratio of refractory to icy material is complex and often under-constrained. This leads to competing interpretations of the same data set. One can find the most recent discussions on this topic in LevasseurRegourd et al. (2018), Fulle et al. (2019), and Choukroun et al. (this book).

\subsection{Porosity}

According to Eq. (3), high porosity reduces the strength with $(1-p)$. Measurements on comet $67 \mathrm{P}$ (Pätzold et al. 2016) show a high porosity $p>70 \%$. Thus the strength is reduced to less than $30 \%$ of nonporous granular matter.

\subsection{Grains Sizes}

The cohesive strength $Y$ in a layer of equally sized spheres goes down with increasing radius of the grains (Eq. (3)). Thus an arrangements of larger grains can be more effectively cracked by the gas than a structure of small grains. This was already pointed out by Kuehrt and Keller (1994) and discussed later in much more detail by Gundlach et al. (2015). When assuming a surface energy of $0.025 \mathrm{~J} \mathrm{~m}^{-2}$, valid for quartz, a porosity of $75 \%$ and four contacts per grain Eq. (3) provides a strength $Y \simeq 0.02 / R$ Pa. The cohesion between $1 \mathrm{~cm}$ grains is in this model $2 \mathrm{~Pa}$, between dm-sized grains $0.2 \mathrm{~Pa}$, which may be low enough to be torn off by gas pressure. For such large grains - that in terms of cometary structures will present hierarchically built clusters of grains-gravity is the limiting factor. In situ observations (Mottola et al. 2015; Ott et al. 2017) and laboratory measurements (Gundlach et al. 2015) have confirmed that $\mathrm{mm}$ to $\mathrm{dm}$ grains that can be easily removed against cohesion and gravity and are common on the surface of comet 67P.

\subsection{Grain Size Distribution}

A further way to build clusters of low cohesion is a mixture of grains of different sizes. In such a structure (Fig. 4) with $R_{2} \ll R_{1}$ the cohesive forces are controlled by the small (black) grains but the gas pressure can act on the cross section of the large (blue) spheres with $R_{2}$. The red dashed line marks a weakly bound cluster and Eqs. (1) and (2) yield:

$$
F_{c}=3 \pi \gamma R_{2}
$$


Fig. 4 Example arrangement of spheres of different sizes in a porous structure. The dashed red line shows a case of a cluster with a weak relative strength

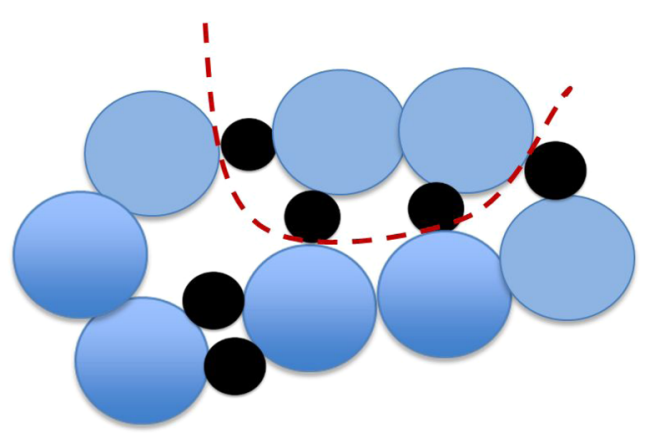

Fig. 5 SEM images at magnification of 10000 of a sample with $\mu$ m-sized $\mathrm{Al}$ particles and R972 nanoparticles for reduction of cohesiveness. Adapted from Chen et al. (2010)

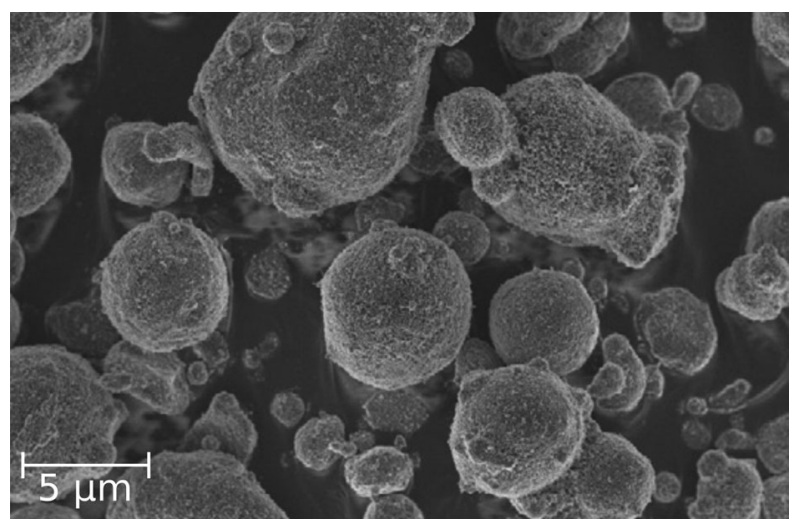

The drag force is given by

$$
F_{d}=\frac{1}{2} \rho v^{2} C_{d} \pi R_{1}^{2}
$$

where $C_{d}$ is the drag coefficient, $v$ the gas velocity, $\rho$ the gas density. Consequently, the ratio of drag force and cohesive binding force is $F_{d} / F_{c} \propto R_{1}^{2} / R_{2}$. It is enhanced by a factor of 1000 when having a mixture of mm- with $\mu \mathrm{m}$ - or $\mu \mathrm{m}$ - with nm-particles compared to only monodisperse $\mathrm{mm}$ - or $\mu \mathrm{m}$-grains, respectively.

When there is a closed line (or surface in 3D) where the local ratio $F_{d} / F_{c}$ is larger than 1 the cluster that is coincidentally formed by this surface can escape by the gas pressure.

An analogous effect can be reached by particles with a rough surface. It should be noted that the described method to reduce the cohesion is known as "dry particle coating" (Fig. 5) in technical applications on Earth (Chen et al. 2010) and is e.g. applied to improve the flow properties of printer toner. More detailed numerical simulations and lab measurements are necessary to quantify this scenario.

For sure the real comet is much more complex in structure than what is shown in Fig. 4 but this is valid approximation. Indeed, atomic force microscope images from the MIDAS instrument on board Rosetta revealed grains with an irregular structure (Fig. 6), which can produce quite weak cohesive bounds, locally, as discussed above.

It should be mentioned that the conditions of such weak structures are mainly given near the surface where most of the ice that clues the dust effectively together by sintering effects has already gone. Therefore, the measured strengths of some Pa to kPa must not be in contradiction to a very low cohesive path $(<1 \mathrm{~Pa})$ along a given area in a surface layer. 
Fig. 6 (a) Crop of a MIDAS post-processed topographic image (b) Corresponding area to the white frame in (a), showing all identified subunits of particle as $2 \mathrm{D}$ projection. Adapted from Mannel et al. (2016) (a)

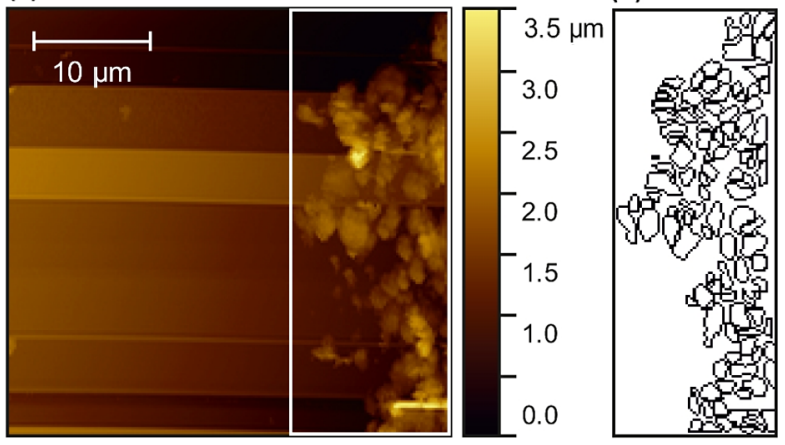

\subsection{Escaping the "Activity Paradox"}

In summary, based on the concepts described here, we argue that the activity paradox can be addressed in two ways:

1. If the dust to ice volume ratio is $<1$ dust grains are isolated in the ice matrix and cannot touch each other to form a cohesive and maybe permanent dust mantle that quenches activity. Independently on the strength of the layer the dust can be removed when the ice is sublimating. However this scenario can only work when the dust/ice mass ratio is small and each dust grain is immediately removed by the gas.

2. Even at a high dust/ice ration physical properties and the microstructure of the cometary surface layer result in local cohesive weaknesses that allow the gas pressure to clear the surface from dust. If irregular clusters at the surface are formed by chance that can be intrinsically stronger than the gas pressure but have weak bonds among themselves they can be lifted off even by low gas pressure. Besides such a zone of low local strength the clusters must be small enough to be removable against gravity. However, this limit is near perihelion in the meter range.

More detailed numerical simulations and lab measurements are necessary to quantify these scenarios. In that context, future sample return missions like CAESAR (NASA New Frontier proposal) will be critical in assessing the real nature of cometary grains and their cohesion.

\section{Conclusion}

This chapter has reviewed what is currently known about local manifestations of cometary activity. We presented a revised nomenclature of active features, recommending the usage of descriptive but neutral terms to describe physical phenomenon we observe but do not yet understand. Throughout the paper, we use jet-like feature when referring to perennial collimated streams, and transient plumes for sudden events.

We listed historical in-situ observations of cometary activity over a 30 years period, from the Giotto mission in 1984 to Rosetta in 2014-2016.

Finally, we dwelved into the "activity paradox", which is that current models of dust release and subsequent acceleration by gas streams formed by sublimating volatiles are not sufficient to explain our observations. While we do not have an answer to this conundrum, we do propose several ways forward, to be investigated further. 
Acknowledgements This project has received funding from the European Union's Horizon 2020 research and innovation programme under grant agreement No. 686709 (MiARD). This work was supported by the Swiss State Secretariat for Education, Research and Innovation (SERI) under contract number 16.0008-2. The opinions expressed and arguments employed herein do not necessarily reflect the official view of the Swiss Government. This research has made use of NASA's Astrophysics Data System Bibliographic Services.

Publisher's Note Springer Nature remains neutral with regard to jurisdictional claims in published maps and institutional affiliations.

\section{References}

J. Agarwal, M.F. A’Hearn, J.B. Vincent, C. Güttler, S. Höfner, H. Sierks, C. Tubiana, C. Barbieri, P.L. Lamy, R. Rodrigo, D. Koschny, H. Rickman, M.A. Barucci, J.L. Bertaux, I. Bertini, S. Boudreault, G. Cremonese, V. Da Deppo, B. Davidsson, S. Debei, M. De Cecco, J. Deller, S. Fornasier, M. Fulle, A. Gicquel, O. Groussin, P.J. Gutiérrez, M. Hofmann, S.F. Hviid, W.H. Ip, L. Jorda, H.U. Keller, J. Knollenberg, J.R. Kramm, E. Kührt, M. Küppers, L.M. Lara, M. Lazzarin, J.J. Lopez Moreno, F. Marzari, G. Naletto, N. Oklay, X. Shi, N. Thomas, Acceleration of individual, decimetre-sized aggregates in the lower coma of comet 67P/Churyumov-Gerasimenko. Mon. Not. R. Astron. Soc. 462, S78-S88 (2016). https://doi.org/10.1093/mnras/stw2179. arXiv:1608.07933

M.F. A'Hearn, M.J.S. Belton, W.A. Delamere, L.M. Feaga, D. Hampton, J. Kissel, K.P. Klaasen, L.A. McFadden, K.J. Meech, H.J. Melosh, P.H. Schultz, J.M. Sunshine, P.C. Thomas, J. Veverka, D.D. Wellnitz, D.K. Yeomans, S. Besse, D. Bodewits, T.J. Bowling, B.T. Carcich, S.M. Collins, T.L. Farnham, O. Groussin, B. Hermalyn, M.S. Kelley, M.S. Kelley, J.Y. Li, D.J. Lindler, C.M. Lisse, S.A. McLaughlin, F. Merlin, S. Protopapa, J.E. Richardson, J.L. Williams, EPOXI at comet Hartley 2. Science 332, 1396 (2011). https://doi.org/10.1126/science.1204054

N. Attree, O. Groussin, L. Jorda, D. Nébouy, N. Thomas, Y. Brouet, E. Kührt, F. Preusker, F. Scholten, J. Knollenberg, P. Hartogh, H. Sierks, C. Barbieri, P. Lamy, R. Rodrigo, D. Koschny, H. Rickman, H.U. Keller, M.F. A'Hearn, A.T. Auger, M.A. Barucci, J.L. Bertaux, I. Bertini, D. Bodewits, S. Boudreault, G. Cremonese, V. Da Deppo, B. Davidsson, S. Debei, M. De Cecco, J. Deller, M.R. ElMaarry, S. Fornasier, M. Fulle, P.J. Gutiérrez, C. Güttler, S. Hviid, W.H. Ip, G. Kovacs, J.R. Kramm, M. Küppers, L.M. Lara, M. Lazzarin, J.J. Lopez Moreno, S. Lowry, S. Marchi, F. Marzari, S. Mottola, G. Naletto, N. Oklay, M. Pajola, I. Toth, C. Tubiana, J.B. Vincent, X. Shi, Tensile strength of 67P/Churyumov-Gerasimenko nucleus material from overhangs. Astron. Astrophys. 611, A33 (2018a). https://doi.org/10.1051/0004-6361/201732155. arXiv:1712.07508

N. Attree, O. Groussin, L. Jorda, S. Rodionov, A.T. Auger, N. Thomas, Y. Brouet, O. Poch, E. Kührt, M. Knapmeyer, F. Preusker, F. Scholten, J. Knollenberg, S. Hviid, P. Hartogh, Thermal fracturing on comets. Applications to 67P/Churyumov-Gerasimenko. Astron. Astrophys. 610, A76 (2018b). https://doi.org/10.1051/0004-6361/201731937. arXiv:1711.09809

M.J.S. Belton, Cometary activity, active areas, and a mechanism for collimated outflows on 1P, 9P, 19P, and 81P. Icarus 210, 881-897 (2010)

M.J.S. Belton, The origin of pits on 9P/Tempel 1 and the geologic signature of outbursts in Stardust-NExT images. Icarus 222, 477-486 (2013)

M.J.S. Belton, B.E.A. Mueller, W.H. Julian, A.J. Anderson, The spin state and homogeneity of comet Halley's nucleus. Icarus 93, 183-193 (1991)

J. Blum, B. Gundlach, S. Mühle, J.M. Trigo-Rodriguez, Comets formed in solar-nebula instabilities!-An experimental and modeling attempt to relate the activity of comets to their formation process. Icarus 235, 156-169 (2014). https://doi.org/10.1016/j.icarus.2014.03.016. arXiv:1403.2610

D. Bodewits, L.M. Lara, M.F. A’Hearn, F. La Forgia, A. Gicquel, G. Kovacs, J. Knollenberg, M. Lazzarin, Z.Y. Lin, X. Shi, C. Snodgrass, C. Tubiana, H. Sierks, C. Barbieri, P.L. Lamy, R. Rodrigo, D. Koschny, H. Rickman, H.U. Keller, M.A. Barucci, J.L. Bertaux, I. Bertini, S. Boudreault, G. Cremonese, V. Da Deppo, B. Davidsson, S. Debei, M. De Cecco, S. Fornasier, M. Fulle, O. Groussin, P.J. Gutiérrez, C. Güttler, S.F. Hviid, W.H. Ip, L. Jorda, J.R. Kramm, E. Kührt, M. Küppers, J.J. López-Moreno, F. Marzari, G. Naletto, N. Oklay, N. Thomas, I. Toth, J.B. Vincent, Changes in the physical environment of the inner coma of 67P/Churyumov-Gerasimenko with decreasing heliocentric distance. Astron. J. 152, 130 (2016). https://doi.org/10.3847/0004-6256/152/5/130. arXiv: 1607.05632

H. Boehnhardt, E. Pompei, G.P. Tozzi, O. Hainaut, N. Ageorges, S. Bagnulo, L. Barrera, T. Bonev, H.U. Käufl, F. Kerber, G. Locurto, O. Marco, E. Pantin, H. Rauer, I. Saviane, F. Selman, C. Sterken, M. Weiler, Broad- and narrowband visible imaging of comet 9P/Tempel 1 at ESO around the time of the Deep Impact event. Astron. Astrophys. 470, 1175-1183 (2007). https://doi.org/10.1051/ 0004-6361:20066577 
D.C. Boice, L.A. Soderblom, D.T. Britt, R.H. Brown, B.R. Sandel, R.V. Yelle, B.J. Buratti, M.D. Hicks, R.M. Nelson, M.D. Rayman, J. Oberst, N. Thomas, The Deep Space 1 encounter with comet 19p/Borrelly. Earth Moon Planets 89, 301-324 (2002). https://doi.org/10.1023/A:1021519124588

M. Bruck Syal, P.H. Schultz, J.M. Sunshine, M.F. A'Hearn, T.L. Farnham, D.S.P. Dearborn, Geologic control of jet formation on comet 103P/Hartley 2. Icarus 222, 610 (2013)

F. Capaccioni, A. Coradini, G. Filacchione, S. Erard, G. Arnold, P. Drossart, M.C. De Sanctis, D. BockeleeMorvan, M.T. Capria, F. Tosi, C. Leyrat, B. Schmitt, E. Quirico, P. Cerroni, V. Mennella, A. Raponi, M. Ciarniello, T. McCord, L. Moroz, E. Palomba, E. Ammannito, M.A. Barucci, G. Bellucci, J. Benkhoff, J.P. Bibring, A. Blanco, M. Blecka, R. Carlson, U. Carsenty, L. Colangeli, M. Combes, M. Combi, J. Crovisier, T. Encrenaz, C. Federico, U. Fink, S. Fonti, W.H. Ip, P. Irwin, R. Jaumann, E. Kuehrt, Y. Langevin, G. Magni, S. Mottola, V. Orofino, P. Palumbo, G. Piccioni, U. Schade, F. Taylor, D. Tiphene, G.P. Tozzi, P. Beck, N. Biver, L. Bonal, J.P. Combe, D. Despan, E. Flamini, S. Fornasier, A. Frigeri, D. Grassi, M. Gudipati, A. Longobardo, K. Markus, F. Merlin, R. Orosei, G. Rinaldi, K. Stephan, M. Cartacci, A. Cicchetti, S. Giuppi, Y. Hello, F. Henry, S. Jacquinod, R. Noschese, G. Peter, R. Politi, J.M. Reess, A. Semery, The organic-rich surface of comet 67P/Churyumov-Gerasimenko as seen by VIRTIS/Rosetta. Science 347(1), aaa0628 (2015). https://doi.org/10.1126/science.aaa0628

W.E. Celnik, T. Schmidt-Kaler, Structure and dynamics of plasma-tail condensations of comet P/Halley 1986 and inferences on the structure and activity of the cometary nucleus. Astron. Astrophys. 187, 233-248 (1987)

Y. Chen, L. Jallo, M.A. Quintanilla, R. Dave, Characterization of particle and bulk level cohesion reduction of surface modified fine aluminum powders. Colloids Surf. A, Physicochem. Eng. Asp. 361(1), 66-80 (2010). https://doi.org/10.1016/j.colsurfa.2010.03.015. http://www.sciencedirect.com/science/ article/pii/S0927775710001895

A. Chokshi, A.G.G.M. Tielens, D. Hollenbach, Dust coagulation. Astrophys. J. 407, 806-819 (1993). https://doi.org/10.1086/172562

M. Choukroun, S. Keihm, F.P. Schloerb, S. Gulkis, E. Lellouch, Dark side of comet 67P/ChuryumovGerasimenko in Aug.-Oct. 2014. MIRO/Rosetta continuum observations of polar night in the southern regions. Astron. Astrophys. 583, A28 (2015). https://doi.org/10.1051/0004-6361/201526181

J.F. Crifo, A.V. Rodionov, The dependence of the circumnuclear coma structure on the properties of the nucleus. Icarus 129, 72-93 (1997). https://doi.org/10.1006/icar.1997.5714

J.F. Crifo, A.V. Rodionov, K. Szegö, M. Fulle, Challenging a paradigm: do we need active and inactive areas to account for near-nuclear jet activity? Earth Moon Planets 90, 227-238 (2002)

J.F. Crifo, G.A. Loukianov, A.V. Rodionov, V.V. Zakharov, Direct Monte Carlo and multifluid modeling of the circumnuclear dust coma. Spherical grain dynamics revisited. Icarus 176, 192-219 (2005)

M.C. De Sanctis, F. Capaccioni, M. Ciarniello, G. Filacchione, M. Formisano, S. Mottola, A. Raponi, F. Tosi, D. Bockelée-Morvan, S. Erard, C. Leyrat, B. Schmitt, E. Ammannito, G. Arnold, M.A. Barucci, M. Combi, M.T. Capria, P. Cerroni, W.H. Ip, E. Kuehrt, T.B. McCord, E. Palomba, P. Beck, E. Quirico (VIRTIS Team), G. Piccioni, G. Bellucci, M. Fulchignoni, R. Jaumann, K. Stephan, A. Longobardo, V. Mennella, A. Migliorini, J. Benkhoff, J.P. Bibring, A. Blanco, M. Blecka, R. Carlson, U. Carsenty, L. Colangeli, M. Combes, J. Crovisier, P. Drossart, T. Encrenaz, C. Federico, U. Fink, S. Fonti, P. Irwin, Y. Langevin, G. Magni, L. Moroz, V. Orofino, U. Schade, F. Taylor, D. Tiphene, G.P. Tozzi, N. Biver, L. Bonal, J.P. Combe, D. Despan, E. Flamini, S. Fornasier, A. Frigeri, D. Grassi, M.S. Gudipati, F. Mancarella, K. Markus, F. Merlin, R. Orosei, G. Rinaldi, M. Cartacci, A. Cicchetti, S. Giuppi, Y. Hello, F. Henry, S. Jacquinod, J.M. Rees, R. Noschese, R. Politi, G. Peter, The diurnal cycle of water ice on comet 67P/Churyumov-Gerasimenko. Nature 525, 500 (2015)

M. Delbo, G. Libourel, J. Wilkerson, N. Murdoch, P. Michel, K.T. Ramesh, C. Ganino, C. Verati, S. Marchi, Thermal fatigue as the origin of regolith on small asteroids. Nature 508, 233-236 (2014). https://doi.org/10.1038/nature13153

M.R. El-Maarry, O. Groussin, N. Thomas, M. Pajola, A.T. Auger, B. Davidsson, X. Hu, S.F. Hviid, J. Knollenberg, C. Güttler, C. Tubiana, S. Fornasier, C. Feller, P. Hasselmann, J.B. Vincent, H. Sierks, C. Barbieri, P. Lamy, R. Rodrigo, D. Koschny, H.U. Keller, H. Rickman, M.F. A'Hearn, M.A. Barucci, J.L. Bertaux, I. Bertini, S. Besse, D. Bodewits, G. Cremonese, V. Da Deppo, S. Debei, M. De Cecco, J. Deller, J.D.P. Deshapriya, M. Fulle, P.J. Gutierrez, M. Hofmann, W.H. Ip, L. Jorda, G. Kovacs, J.R. Kramm, E. Kührt, M. Küppers, L.M. Lara, M. Lazzarin, Surface changes on comet 67P/Churyumov-Gerasimenko suggest a more active past. Science 355, 1392-1395 (2017). https://doi.org/10.1126/science.aak9384

T.L. Farnham, Dust jet activity in comet 103P/Hartley 2, in Asteroids, Comets, Meteors, vol. 1667 (2012), p. 6241

T.L. Farnham, A.L. Cochran, A McDonald Observatory study of comet 19P/Borrelly: placing the Deep Space 1 observations into a broader context. Icarus 160, 398-418 (2002). https://doi.org/ 10.1006/icar.2002.6969. arXiv:astro-ph/0208445 
T.L. Farnham, D.G. Schleicher, Physical and compositional studies of comet 81P/Wild 2 at multiple apparitions. Icarus 173, 533-558 (2005). https://doi.org/10.1016/j.icarus.2004.08.021

T.L. Farnham, B.E.A. Mueller, M.M. Knight, N.H. Samarasinha, Narrowband observations of comet Tempel 1 in support of the Deep Impact mission, in AAS/Division for Planetary Sciences Meeting Abstracts, vol. 37 (2005) p. 43.05

T.L. Farnham, D.D. Wellnitz, D.L. Hampton, J.Y. Li, J.M. Sunshine, O. Groussin, L.A. McFadden, C.J. Crockett, M.F. A'Hearn, M.J.S. Belton, P. Schultz, C.M. Lisse, Dust coma morphology in the Deep Impact images of comet 9P/Tempel 1. Icarus 187, 26-40 (2007). 10.1016/j.icarus.2006.10.036

T.L. Farnham, N.H. Samarasinha, B.E.A. Mueller, Comet 81P/Wild, in International Astronomical Union Circular, vol. 9138 (2010), p. 2

T.L. Farnham, S. Besse, L.M. Feaga, J.M. Sunshine, M.F. A'Hearn, D. Lindler, D. Bodewits, C.M. Lisse, M.J.S. Belton (Dixi Team), Jet activity in comet 103P/Hartley 2 as observed by the Deep Impact Spacecraft, in Lunar and Planetary Science Conference (2011), p. 2160

T.L. Farnham, D. Bodewits, J.Y. Li, J. Veverka, P. Thomas, M.J.S. Belton, Connections between the jet activity and surface features on comet 9P/Tempel 1. Icarus 222, 540-549 (2013). https://doi.org/ 10.1016/j.icarus.2012.06.019

L.M. Feaga, M.F. A'Hearn, J.M. Sunshine, O. Groussin, T.L. Farnham, Asymmetries in the distribution of $\mathrm{H}_{2} \mathrm{O}$ and $\mathrm{CO}_{2}$ in the inner coma of comet 9P/Tempel 1 as observed by Deep Impact. Icarus 190, 345356 (2007)

M.L. Finson, R.F. Probstein, A theory of dust comets. I. Model and equations. Astrophys. J. 154, 353-380 (1968)

S. Fornasier, C. Feller, J.C. Lee, S. Ferrari, M. Massironi, P.H. Hasselmann, J.D.P. Deshapriya, M.A. Barucci, M.R. El-Maarry, L. Giacomini, S. Mottola, H.U. Keller, W.H. Ip, Z.Y. Lin, H. Sierks, C. Barbieri, P.L. Lamy, R. Rodrigo, D. Koschny, H. Rickman, J. Agarwal, M. A'Hearn, J.L. Bertaux, I. Bertini, G. Cremonese, V. Da Deppo, B. Davidsson, S. Debei, M. De Cecco, J. Deller, M. Fulle, O. Groussin, P.J. Gutierrez, C. Güttler, M. Hofmann, S.F. Hviid, L. Jorda, J. Knollenberg, G. Kovacs, R. Kramm, E. Kührt, M. Küppers, M.L. Lara, M. Lazzarin, J.J. Lopez Moreno, F. Marzari, G. Naletto, N. Oklay, M. Pajola, X. Shi, N. Thomas, I. Toth, C. Tubiana, J.B. Vincent, The highly active Anhur-Bes regions in the 67P/Churyumov-Gerasimenko comet: results from OSIRIS/ROSETTA observations. Mon. Not. R. Astron. Soc. 469, S93-S107 (2017). https://doi.org/10.1093/mnras/stx1275. arXiv:1707.02945

M. Fulle, J. Blum, S.F. Green, B. Gundlach, A. Herique, F. Moreno, S. Mottola, A. Rotundi, C. Snodgrass, The refractory-to-ice mass ratio in comets. Mon. Not. R. Astron. Soc. 482, 3326-3340 (2019). https://doi.org/10.1093/mnras/sty2926

O. Groussin et al., Gravitational slopes, geomorphology and material strengths of the nucleus of comet 67P/Churyumov-Gerasimenko from OSIRIS observations. Astron. Astrophys. (2015). https://doi.org/ 10.1051/0004-6361/201526379

E. Grün, J. Gebhard, A. Bar-Nun, J. Benkhoff, H. Düren, G. Eich, R. Hische, W.F. Huebner, H.U. Keller, G. Klees, H. Kochan, G. Kölzer, H. Kroker, E. Kührt, P. Lämmerzahl, E. Lorenz, W.J. Markiewicz, D. Möhlmann, A. Oehler, J. Scholz, K.J. Seidensticker, K. Roessler, G. Schwehm, G. Steiner, K. Thiel, H. Thomas, Development of a dust mantle on the surface of an insolated ice-dust mixture-results from the KOSI-9 experiment. J. Geophys. Res. 98, 15 (1993). https://doi.org/10.1029/93JE01134

E. Grün, J. Agarwal, N. Altobelli, K. Altwegg, M.S. Bentley, N. Biver, V. Della Corte, N. Edberg, P.D. Feldman, M. Galand, B. Geiger, C. Götz, B. Grieger, C. Güttler, P. Henri, M. Hofstadter, M. Horanyi, E. Jehin, H. Krüger, S. Lee, T. Mannel, E. Morales, O. Mousis, M. Müller, C. Opitom, A. Rotundi, R. Schmied, F. Schmidt, H. Sierks, C. Snodgrass, R.H. Soja, M. Sommer, R. Srama, C.Y. Tzou, J.B. Vincent, P. Yanamandra-Fisher, M.F. A'Hearn, A.I. Erikson, C. Barbieri, M.A. Barucci, J.L. Bertaux, I. Bertini, J. Burch, L. Colangeli, G. Cremonese, V. Da Deppo, B. Davidsson, S. Debei, M. De Cecco, J. Deller, L.M. Feaga, M. Ferrari, S. Fornasier, M. Fulle, A. Gicquel, M. Gillon, S.F. Green, O. Groussin, P.J. Gutiérrez, M. Hofmann, S.F. Hviid, W.H. Ip, S. Ivanovski, L. Jorda, H.U. Keller, M.M. Knight, J. Knollenberg, D. Koschny, J.R. Kramm, E. Kührt, M. Küppers, P.L. Lamy, L.M. Lara, M. Lazzarin, J.J. Lòpez-Moreno, J. Manfroid, E.M. Epifani, F. Marzari, G. Naletto, N. Oklay, P. Palumbo, J.W. Parker, H. Rickman, R. Rodrigo, J. Rodrìguez, E. Schindhelm, X. Shi, R. Sordini, A.J. Steffl, S.A. Stern, N. Thomas, C. Tubiana, H.A. Weaver, P. Weissman, V.V. Zakharov, M.G.G.T. Taylor, The 2016 Feb 19 outburst of comet 67P/CG: an ESA Rosetta multi-instrument study. Mon. Not. R. Astron. Soc. 462, S220-S234 (2016). https://doi.org/10.1093/mnras/stw2088

B. Gundlach, J. Blum, H.U. Keller, Y.V. Skorov, What drives the dust activity of comet 67P/ChuryumovGerasimenko? Astron. Astrophys. 583, A12 (2015). https://doi.org/10.1051/0004-6361/201525828. arXiv: 1506.08545

C. Güttler, M. Krause, R.J. Geretshauser, R. Speith, J. Blum, The physics of protoplanetesimal dust agglomerates. IV. Toward a dynamical collision model. Astrophys. J. 701, 130-141 (2009). https://doi.org/10.1088/0004-637X/701/1/130. arXiv:0906.0088 
K.C. Hansen, K. Altwegg, J.J. Berthelier, A. Bieler, N. Biver, D. Bockelée-Morvan, U. Calmonte, F. Capaccioni, M.R. Combi, J. de Keyser, B. Fiethe, N. Fougere, S.A. Fuselier, S. Gasc, T.I. Gombosi, Z. Huang, L. Le Roy, S. Lee, H. Nilsson, M. Rubin, Y. Shou, C. Snodgrass, V. Tenishev, G. Toth, C.Y. Tzou, C.S. Wedlund (Rosina Team), Evolution of water production of 67P/Churyumov-Gerasimenko: an empirical model and a multi-instrument study. Mon. Not. R. Astron. Soc. 462, S491-S506 (2016). https://doi.org/10.1093/mnras/stw2413

M. Hässig, K. Altwegg, H. Balsiger, A. Bar-Nun, J.J. Berthelier, A. Bieler, P. Bochsler, C. Briois, U. Calmonte, M. Combi, J. De Keyser, P. Eberhardt, B. Fiethe, S.A. Fuselier, M. Galand, S. Gasc, T.I. Gombosi, K.C. Hansen, A. Jäckel, H.U. Keller, E. Kopp, A. Korth, E. Kührt, L. Le Roy, U. Mall, B. Marty, O. Mousis, E. Neefs, T. Owen, H. Rème, M. Rubin, T. Sémon, C. Tornow, C.Y. Tzou, J.H. Waite, P. Wurz, Time variability and heterogeneity in the coma of 67P/Churyumov-Gerasimenko. Science 347, aaa0276 (2015). https://doi.org/10.1126/science.aaa0276

B. Hermalyn, T.L. Farnham, S.M. Collins, M.S. Kelley, M.F. A'Hearn, D. Bodewits, B. Carcich, D.J. Lindler, C. Lisse, K. Meech, P.H. Schultz, P.C. Thomas, The detection, localization, and dynamics of large icy particles surrounding comet 103P/Hartley 2. Icarus 222, 625-633 (2013). https://doi.org/10.1016/j.icarus.2012.09.030

M. Horányi, J.R. Szalay, S. Kempf, J. Schmidt, E. Grün, R. Srama, Z. Sternovsky, A permanent, asymmetric dust cloud around the Moon. Nature 522, 324-326 (2015). https://doi.org/10.1038/nature14479

K.L. Johnson, K. Kendall, A.D. Roberts, Surface energy and the contact of elastic solids. Proc. R. Soc. Lond. Ser. A 324, 301-313 (1971). https://doi.org/10.1098/rspa.1971.0141

H.U. Keller, W.A. Delamere, H.J. Reitsema, W.F. Huebner, H.U. Schmidt, Comet P/Halley's nucleus and its activity. Astron. Astrophys. 187, 807-823 (1987)

H.U. Keller, S. Mottola, B. Davidsson, S.E. Schröder, Y. Skorov, E. Kührt, O. Groussin, M. Pajola, S.F. Hviid, F. Preusker, F. Scholten, M.F. A'Hearn, H. Sierks, C. Barbieri, P. Lamy, R. Rodrigo, D. Koschny, H. Rickman, M.A. Barucci, J.L. Bertaux, I. Bertini, G. Cremonese, V. Da Deppo, S. Debei, M. De Cecco, S. Fornasier, M. Fulle, P.J. Gutiérrez, W.H. Ip, L. Jorda, J. Knollenberg, J.R. Kramm, M. Küppers, L.M. Lara, M. Lazzarin, J.J. Lopez Moreno, F. Marzari, H. Michalik, G. Naletto, L. Sabau, N. Thomas, J.B. Vincent, K.P. Wenzel, J. Agarwal, C. Güttler, N. Oklay, C. Tubiana, Insolation, erosion, and morphology of comet 67P/Churyumov-Gerasimenko. Astron. Astrophys. 583, A34 (2015). https://doi.org/10.1051/0004-6361/201525964

M.S. Kelley, D.J. Lindler, D. Bodewits, M.F. A'Hearn, C.M. Lisse, L. Kolokolova, J. Kissel, B. Hermalyn, A distribution of large particles in the coma of comet 103P/Hartley 2. Icarus 222, 634-652 (2013). https://doi.org/10.1016/j.icarus.2012.09.037. arXiv:1304.4204

J. Knollenberg, A mini outburst from the nightside of comet P67/Churyumov-Gerasimenko observed by the OSIRIS camera on Rosetta. Astron. Astrophys. (2015)

J. Knollenberg, E. Kührt, H.U. Keller, Interpretation of HMC images by a combined thermal and gasdynamic model. Earth Moon Planets 72, 103-112 (1996). https://doi.org/10.1007/BF00117509

K.J. Kossacki, T. Spohn, A. Hagermann, E. Kaufmann, E. Kührt, Comet 67P/Churyumov-Gerasimenko: hardening of the sub-surface layer. Icarus 260, 464-474 (2015). https://doi.org/10.1016/j.icarus.2015.07.024

T. Kramer, M. Noack, On the origin of inner coma structures observed by Rosetta during a diurnal rotation of comet 67P/Churyumov-Gerasimenko. Astrophys. J. Lett. 823, L11 (2016). https://doi.org/10.3847/2041-8205/823/1/L11. arXiv:1607.03825

T. Kramer, M. Läuter, M. Rubin, K. Altwegg, Seasonal changes of the volatile density in the coma and on the surface of comet 67P/Churyumov-Gerasimenko. Mon. Not. R. Astron. Soc. 469, S20-S28 (2017). https://doi.org/10.1093/mnras/stx866. arXiv:1704.03874

E. Kuehrt, H.U. Keller, The formation of cometary surface crusts. Icarus 109, 121-132 (1994). https://doi.org/10.1006/icar.1994.1080

I.L. Lai, W.H. Ip, J.C. Lee, Z.Y. Lin, J.B. Vincent, P. Hartogh, H. Sierks, C. Barbieri, P.L. Lamy, H. Rickman, H.U. Keller, Mapping of the source regions of the dust jets on comet 67P/Churyumov-Gerasimenko, in AGU Fall Meeting Abstracts P43A-2087 (2016)

L.M. Lara, H. Böhnhardt, R. Gredel, P.J. Gutiérrez, J.L. Ortiz, R. Rodrigo, M.J. Vidal-Nuñez, Pre-impact monitoring of comet 9P/Tempel 1, the Deep Impact target. Astron. Astrophys. 465, 1151-1157 (2006)

L.M. Lara et al., Large-scale dust jets in the coma of 67P/Churyumov-Gerasimenko as seen by the OSIRIS instrument onboard Rosetta. Astron. Astrophys. (2015). https://doi.org/10.1051/0004-6361/201526103

S. Larson, Z. Sekanina, D. Levy, S. Tapia, M. Senay, Comet p/Halley near nucleus phenomena in 1986. Astron. Astrophys. 187, 639 (1987)

A.C. Levasseur-Regourd, J. Agarwal, H. Cottin, C. Engrand, G. Flynn, M. Fulle, T. Gombosi, Y. Langevin, J. Lasue, T. Mannel, S. Merouane, O. Poch, N. Thomas, A. Westphal, Cometary dust. Space Sci. Rev. 214, 64 (2018). https://doi.org/10.1007/s11214-018-0496-3

Z.Y. Lin et al., Morphology and dynamics of jets of comet 67P/Churyumov-Gerasimenko: early phase development. Astron. Astrophys. (2015). https://doi.org/10.1051/0004-6361/201525961 
Z.Y. Lin, I.L. Lai, C.C. Su, W.H. Ip, J.C. Lee, J.S. Wu, J.B. Vincent, F. La Forgia, H. Sierks, C. Barbieri, P.L. Lamy, R. Rodrigo, D. Koschny, H. Rickman, H.U. Keller, J. Agarwal, M.F. A’Hearn, M.A. Barucci, J.L. Bertaux, I. Bertini, D. Bodewits, G. Cremonese, V. Da Deppo, B. Davidsson, S. Debei, M. De Cecco, S. Fornasier, M. Fulle, O. Groussin, P.J. Gutiérrez, C. Güttler, S.F. Hviid, L. Jorda, J. Knollenberg, G. Kovacs, J.R. Kramm, E. Kührt, M. Küppers, L.M. Lara, M. Lazzarin, J.J. López-Moreno, S. Lowry, F. Marzari, H. Michalik, S. Mottola, G. Naletto, N. Oklay, M. Pajola, A. Rozek, N. Thomas, C. Tubiana, Observations and analysis of a curved jet in the coma of comet 67P/Churyumov-Gerasimenko. Astron. Astrophys. 588, L3 (2016). https://doi.org/10.1051/0004-6361/201527784

T. Mannel, M.S. Bentley, R. Schmied, H. Jeszenszky, A.C. Levasseur-Regourd, J. Romstedt, K. Torkar, Fractal cometary dust-a window into the early Solar system. Mon. Not. R. Astron. Soc. 462, S304S311 (2016). https://doi.org/10.1093/mnras/stw2898

U. Marboeuf, B. Schmitt, J.M. Petit, O. Mousis, N. Fray, A cometary nucleus model taking into account all phase changes of water ice: amorphous, crystalline, and clathrate. Astron. Astrophys. 542, A82 (2012). https://doi.org/10.1051/0004-6361/201118176

R. Marschall, S. Mottola, C.C. Su, Y. Liao, M. Rubin, J.S. Wu, N. Thomas, K. Altwegg, H. Sierks, W.H. Ip, H.U. Keller, J. Knollenberg, E. Kührt, I.L. Lai, Y. Skorov, L. Jorda, F. Preusker, F. Scholten, J.B. Vincent (Osiris Team Rosina Team), Cliffs versus plains: can ROSINA/COPS and OSIRIS data of comet 67P/Churyumov-Gerasimenko in autumn 2014 constrain inhomogeneous outgassing? Astron. Astrophys. 605, A112 (2017). https://doi.org/10.1051/0004-6361/201730849

R. Miles, Heat of solution: a new source of thermal energy in the subsurface of cometary nuclei and the gas-exsolution mechanism driving outbursts of comet 29P/Schwassmann-Wachmann and other comets. Icarus 272, 356-386 (2016). https://doi.org/10.1016/j.icarus.2015.12.053

S. Mottola, G. Arnold, H.G. Grothues, R. Jaumann, H. Michaelis, G. Neukum, J.P. Bibring, S.E. Schröder, M. Hamm, K.A. Otto, I. Pelivan, G. Proffe, F. Scholten, D. Tirsch, M. Kreslavsky, E. Remetean, F. Souvannavong, B. Dolives, The structure of the regolith on 67P/Churyumov-Gerasimenko from ROLIS descent imaging. Science 349(2), aab0232 (2015). https://doi.org/10.1126/science.aab0232

N. Oklay, Large sub-surface volatile reservoirs of comet 67P, in Lunar and Planetary Science Conference, vol. 49 (2018), p. 1282

N. Oklay, J.B. Vincent, S. Fornasier, M. Pajola, S. Besse, B.J.R. Davidsson, L.M. Lara, S. Mottola, G. Naletto, H. Sierks, A.M. Barucci, F. Scholten, F. Preusker, A. Pommerol, N. Masoumzadeh, M. Lazzarin, C. Barbieri, P.L. Lamy, R. Rodrigo, D. Koschny, H. Rickman, M.F. A'Hearn, J.L. Bertaux, I. Bertini, D. Bodewits, G. Cremonese, V. Da Deppo, S. Debei, M. De Cecco, M. Fulle, O. Groussin, P.J. Gutiérrez, C. Güttler, I. Hall, M. Hofmann, S.F. Hviid, W.H. Ip, L. Jorda, H.U. Keller, J. Knollenberg, G. Kovacs, J.R. Kramm, E. Kührt, M. Küppers, Z.Y. Lin, J.J. Lopez Moreno, F. Marzari, F. Moreno, X. Shi, N. Thomas, I. Toth, C. Tubiana, Variegation of comet 67P/Churyumov-Gerasimenko in regions showing activity. Astron. Astrophys. 586, A80 (2016). https://doi.org/10.1051/0004-6361/201527369

T. Ott, E. Drolshagen, D. Koschny, C. Güttler, C. Tubiana, E. Frattin, J. Agarwal, H. Sierks, I. Bertini, C. Barbieri, P.I. Lamy, R. Rodrigo, H. Rickman, M.F. A'Hearn, M.A. Barucci, J.L. Bertaux, S. Boudreault, G. Cremonese, V. Da Deppo, B. Davidsson, S. Debei, M. De Cecco, J. Deller, C. Feller, S. Fornasier, M. Fulle, B. Geiger, A. Gicquel, O. Groussin, P.J. Gutiérrez, M. Hofmann, S.F. Hviid, W.H. Ip, L. Jorda, H.U. Keller, J. Knollenberg, G. Kovacs, J.R. Kramm, E. Kührt, M. Küppers, L.M. Lara, M. Lazzarin, Z.Y. Lin, J.J. López-Moreno, F. Marzari, S. Mottola, G. Naletto, N. Oklay, M. Pajola, X. Shi, N. Thomas, J.B. Vincent, B. Poppe, Dust mass distribution around comet 67P/Churyumov-Gerasimenko determined via parallax measurements using Rosetta's OSIRIS cameras. Mon. Not. R. Astron. Soc. 469, S276-S284 (2017). https://doi.org/10.1093/mnras/stx1419

M. Pajola, S. Hoefner, J.B. Vincent, O. Nea, The pristine interior of comet 67P revealed by the combined Aswan outburst and cliff collapse. Nature 1, 0092 (2017)

M. Pätzold, T. Andert, M. Hahn, S.W. Asmar, J.P. Barriot, M.K. Bird, B. Häusler, K. Peter, S. Tellmann, E. Grün, P.R. Weissman, H. Sierks, L. Jorda, R. Gaskell, F. Preusker, F. Scholten, A homogeneous nucleus for comet 67P/Churyumov-Gerasimenko from its gravity field. Nature 530, 63-65 (2016). https://doi.org/10.1038/nature16535

S. Protopapa, J.M. Sunshine, L.M. Feaga, M.S.P. Kelley, M.F. A'Hearn, T.L. Farnham, O. Groussin, S. Besse, F. Merlin, J.Y. Li, Water ice and dust in the innermost coma of comet 103P/Hartley 2. Icarus 238, 191204 (2014). https://doi.org/10.1016/j.icarus.2014.04.008. arXiv:1406.3382

A. Rotundi, H. Sierks, V. Della Corte, M. Fulle, P.J. Gutierrez, L. Lara, C. Barbieri, P.L. Lamy, R. Rodrigo, D. Koschny, H. Rickman, H.U. Keller, J.J. López-Moreno, M. Accolla, J. Agarwal, M.F. A’Hearn, N. Altobelli, F. Angrilli, M.A. Barucci, J.L. Bertaux, I. Bertini, D. Bodewits, E. Bussoletti, L. Colangeli, M. Cosi, G. Cremonese, J.F. Crifo, V. Da Deppo, B. Davidsson, S. Debei, M. De Cecco, F. Esposito, M. Ferrari, S. Fornasier, F. Giovane, B. Gustafson, S.F. Green, O. Groussin, E. Grün, C. Güttler, M.L. Herranz, S.F. Hviid, W. Ip, S. Ivanovski, J.M. Jerónimo, L. Jorda, J. Knollenberg, R. Kramm, E. Kührt, M. Küppers, M. Lazzarin, M.R. Leese, A.C. López-Jiménez, F. Lucarelli, S.C. Lowry, F. Marzari, E.M. 
Epifani, J.A.M. McDonnell, V. Mennella, H. Michalik, A. Molina, R. Morales, F. Moreno, S. Mottola, G. Naletto, N. Oklay, J.L. Ortiz, E. Palomba, P. Palumbo, J.M. Perrin, J. Rodríguez, L. Sabau, C. Snodgrass, R. Sordini, N. Thomas, C. Tubiana, J.B. Vincent, P. Weissman, K.P. Wenzel, V. Zakharov, J.C. Zarnecki, Dust measurements in the coma of comet 67P/Churyumov-Gerasimenko inbound to the Sun. Science 347, aaa3905 (2015). https://doi.org/10.1126/science.aaa3905

R.Z. Sagdeev, G.A. Avanesov, B.S. Zhukov, I.V. Zenkin, Y.L. Ziman, V.I. Moroz, V.I. Tarnopol'Skij, Photometric characteristics of the nucleus and inner coma of comet Halley. Kosm. Issled. 25, 831-839 (1987)

N.H. Samarasinha, S.M. Larson, Image enhancement techniques for quantitative investigations of morphological features in cometary comae: a comparative study. Icarus 239, 168 (2014)

D.G. Schleicher, Compositional and physical results for Rosetta's new target comet 67P/Churyumov Gerasimenko from narrowband photometry and imaging. Icarus 181, $442-457$ (2006). https://doi.org/10.1016/j.icarus.2005.11.014

R. Schulz, J.A. Stüwe, H. Boehnhardt, W. Gaessler, G.P. Tozzi, Characterization of STARDUST target comet 81P/Wild 2 from 1996 to 1998. Astron. Astrophys. 398, 345-352 (2003). https://doi.org/10.1051/ 0004-6361:20021673

Z. Sekanina, D.E. Brownlee, T.E. Economou, A.J. Tuzzolino, S.F. Green, Modeling the nucleus and jets of comet 81P/Wild 2 based on the Stardust encounter data. Science 304, 1769-1774 (2004)

X. Shi, X. Hu, H. Sierks, C. Güttler, M. A’Hearn, J. Blum, M.R. El-Maarry, E. Kührt, S. Mottola, M. Pajola, N. Oklay, S. Fornasier, C. Tubiana, H.U. Keller, J.B. Vincent, D. Bodewits, S. Höfner, Z.Y. Lin, A. Gicquel, M. Hofmann, C. Barbieri, P.L. Lamy, R. Rodrigo, D. Koschny, H. Rickman, M.A. Barucci, J.L. Bertaux, I. Bertini, G. Cremonese, V. Da Deppo, B. Davidsson, S. Debei, M. De Cecco, M. Fulle, O. Groussin, P.J. Gutiérrez, S.F. Hviid, W.H. Ip, L. Jorda, J. Knollenberg, G. Kovacs, J.R. Kramm, M. Küppers, L.M. Lara, M. Lazzarin, J.J. Lopez-Moreno, F. Marzari, G. Naletto, N. Thomas, Sunset jets observed on comet 67P/Churyumov-Gerasimenko sustained by subsurface thermal lag. Astron. Astrophys. 586, A7 (2016). https://doi.org/10.1051/0004-6361/201527123

X. Shi, X. Hu, S. Mottola, H. Sierks, H.U. Keller, M. Rose, C. Güttler, M. Fulle, S. Fornasier, J. Agarwal, M. Pajola, C. Tubiana, D. Bodewits, C. Barbieri, P.L. Lamy, R. Rodrigo, D. Koschny, M.A. Barucci, J.L. Bertaux, I. Bertini, S. Boudreault, G. Cremonese, V. Da Deppo, B. Davidsson, S. Debei, M. De Cecco, J. Deller, O. Groussin, P.J. Gutiérrez, S.F. Hviid, W.H. Ip, L. Jorda, J. Knollenberg, G. Kovacs, J.R. Kramm, E. Kührt, M. Küppers, L.M. Lara, M. Lazzarin, J.J. Lopez-Moreno, F. Marzari, G. Naletto, N. Oklay, I. Toth, J.B. Vincent, Coma morphology of comet 67P controlled by insolation over irregular nucleus. Nat. Astron. 2, 562-567 (2018). https://doi.org/10.1038/s41550-018-0481-5

Y. Skorov, J. Blum, Dust release and tensile strength of the non-volatile layer of cometary nuclei. Icarus 221, 1 (2012)

Y.V. Skorov, L. Rezac, P. Hartogh, H.U. Keller, Is near-surface ice the driver of dust activity on 67P/Churyumov-Gerasimenko. Astron. Astrophys. 600, A142 (2017). https://doi.org/10.1051/ 0004-6361/201630000

L.A. Soderblom, T.L. Becker, G. Bennett, D.C. Boice, D.T. Britt, R.H. Brown, B.J. Buratti, C. Isbell, B. Giese, T. Hare, M.D. Hicks, E. Howington-Kraus, R.L. Kirk, M. Lee, R.M. Nelson, J. Oberst, T.C. Owen, M.D. Rayman, B.R. Sandel, S.A. Stern, N. Thomas, R.V. Yelle, Observations of comet 19P/Borrelly by the miniature integrated camera and spectrometer aboard Deep Space 1. Science 296, 1087-1091 (2002). https://doi.org/10.1126/science.1069527

J.K. Steckloff, K. Graves, M. Hirabayashi, H.J. Melosh, J.E. Richardson, Rotationally induced surface slopeinstabilities and the activation of $\mathrm{CO}_{2}$ activity on comet 103P/Hartley 2. Icarus 272, 60-69 (2016). https://doi.org/10.1016/j.icarus.2016.02.026. arXiv:1602.08518

P.J. Stooke, A. Abergel, Morphology of the nucleus of comet P/Halley. Astron. Astrophys. 248, 656-668 (1991)

J.M. Sunshine, M.F. A’Hearn, O. Groussin, J.Y. Li, M.J.S. Belton, W.A. Delamere, J. Kissel, K.P. Klaasen, L.A. McFadden, K.J. Meech, H.J. Melosh, P.H. Schultz, P.C. Thomas, J. Veverka, D.K. Yeomans, I.C. Busko, M. Desnoyer, T.L. Farnham, L.M. Feaga, D.L. Hampton, D.J. Lindler, C.M. Lisse, D.D. Wellnitz, Exposed water ice deposits on the surface of comet 9P/Tempel 1. Science 311, 1453-1455 (2006). https://doi.org/10.1126/science.1123632

J.M. Sunshine, O. Groussin, P.H. Schultz, M.F. A'Hearn, L.M. Feaga, T.L. Farnham, K.P. Klaasen, The distribution of water ice in the interior of comet Tempel 1. Icarus 190, 284-294 (2007). https://doi.org/10.1016/j.icarus.2007.04.024

J.M. Sunshine, L.M. Feaga, O. Groussin, S. Protopapa, M.F. A'Hearn, T.L. Farnham, F. Merlin, J.Y. Li, S. Besse, Water ice on comet 103P/Hartley 2, in EPSC-DPS Joint Meeting 2011, vol. 2011 (2011), p. 1345

H. Thomas, L. Ratke, H. Kochan, Crushing strength of porous ice-mineral bodies-relevance for comets. Adv. Space Res. 14, 207-216 (1994). https://doi.org/10.1016/0273-1177(94)90271-2 
P.C. Thomas, J. Veverka, M.J.S. Belton, A. Hidy, M.F. A’Hearn, T.L. Farnham, O. Groussin, J.Y. Li, L.A. McFadden, J. Sunshine, D. Wellnitz, C. Lisse, P. Schultz, K.J. Meech, W.A. Delamere, The shape, topography, and geology of Tempel 1 from Deep Impact observations. Icarus 187, 4-15 (2007)

P. Thomas et al., The nucleus of comet 9P/Tempel 1: shape and geology from two flybys. Icarus 222, $453-466$ (2013)

N. Thomas, B. Davidsson, M.R. El-Maarry, S. Fornasier, L. Giacomini, A.G. Gracia-Berná, S.F. Hviid, W.H. Ip, L. Jorda, H.U. Keller, J. Knollenberg, E. Kührt, F. La Forgia, I.L. Lai, Y. Liao, R. Marschall, M. Massironi, S. Mottola, M. Pajola, O. Poch, A. Pommerol, F. Preusker, F. Scholten, C.C. Su, J.S. Wu, J.B. Vincent, H. Sierks, C. Barbieri, P.L. Lamy, R. Rodrigo, D. Koschny, H. Rickman, M.F. A'Hearn, M.A. Barucci, J.L. Bertaux, I. Bertini, G. Cremonese, V. Da Deppo, S. Debei, M. de Cecco, M. Fulle, O. Groussin, P.J. Gutierrez, J.R. Kramm, M. Küppers, L.M. Lara, M. Lazzarin, J.J. Lopez Moreno, F. Marzari, H. Michalik, G. Naletto, J. Agarwal, C. Güttler, N. Oklay, C. Tubiana, Redistribution of particles across the nucleus of comet 67P/Churyumov-Gerasimenko. Astron. Astrophys. 583, A17 (2015). https://doi.org/10.1051/0004-6361/201526049

C. Tubiana et al., 67P/Churyumov-Gerasimenko: activity between March and June 2014 as observed from Rosetta/OSIRIS. Astron. Astrophys. 573, A62 (2015)

J.B. Vincent, Evolution of cometary surfaces, in Lunar and Planetary Science Conference, vol. 49 (2018), p. 1280

J.B. Vincent, H. Böhnhardt, I. Bertini, L. Lara, M. Küppers, R. Rodrigo, Coma structures in comet 73P/Schwassmann-Wachmann 3, components B and C, between January and May 2006. Earth Moon Planets 106, 27 (2010)

J.B. Vincent, H. Sierks, M. Rose, Y. Skorov, 3D modeling of cometary dust jets, from the nucleus surface to infinity, in EPSC, vol. 2012 (2012), p. 358

J.B. Vincent, D. Bodewits, S. Besse, H. Sierks, C. Barbieri, P. Lamy, R. Rodrigo, D. Koschny, H. Rickman, H.U. Keller, J. Agarwal, M.F. A’Hearn, A.T. Auger, M.A. Barucci, J.L. Bertaux, I. Bertini, C. Capanna, G. Cremonese, V. da Deppo, B. Davidsson, S. Debei, M. de Cecco, M.R. El-Maarry, F. Ferri, S. Fornasier, M. Fulle, R. Gaskell, L. Giacomini, O. Groussin, A. Guilbert-Lepoutre, P. Gutierrez-Marques, P.J. Gutiérrez, C. Güttler, N. Hoekzema, S. Höfner, S.F. Hviid, W.H. Ip, L. Jorda, J. Knollenberg, G. Kovacs, R. Kramm, E. Kührt, M. Küppers, F. La Forgia, L.M. Lara, M. Lazzarin, V. Lee, C. Leyrat, Z.Y. Lin, J.J. Lopez Moreno, S. Lowry, S. Magrin, L. Maquet, S. Marchi, F. Marzari, M. Massironi, H. Michalik, R. Moissl, S. Mottola, G. Naletto, N. Oklay, M. Pajola, F. Preusker, F. Scholten, N. Thomas, I. Toth, C. Tubiana, Large heterogeneities in comet $67 \mathrm{P}$ as revealed by active pits from sinkhole collapse. Nature 523, 63-66 (2015a). https://doi.org/10.1038/nature14564

J.B. Vincent, N. Oklay, S. Marchi, S. Höfner, H. Sierks, Craters on comets. Planet. Space Sci. 107, 53-63 (2015b). https://doi.org/10.1016/j.pss.2014.06.008

J.B. Vincent, M.F. A’Hearn, Z.Y. Lin, M.R. El-Maarry, M. Pajola, H. Sierks, C. Barbieri, P.L. Lamy, R. Rodrigo, D. Koschny, H. Rickman, H.U. Keller, J. Agarwal, M.A. Barucci, J.L. Bertaux, I. Bertini, S. Besse, D. Bodewits, G. Cremonese, V. Da Deppo, B. Davidsson, S. Debei, M. De Cecco, J. Deller, S. Fornasier, M. Fulle, A. Gicquel, O. Groussin, P.J. Gutiérrez, P. Gutiérrez-Marquez, C. Güttler, S. Höfner, M. Hofmann, S.F. Hviid, W.H. Ip, L. Jorda, J. Knollenberg, G. Kovacs, J.R. Kramm, E. Kührt, M. Küppers, L.M. Lara, M. Lazzarin, J.J. Lopez Moreno, F. Marzari, M. Massironi, S. Mottola, G. Naletto, N. Oklay, F. Preusker, F. Scholten, X. Shi, N. Thomas, I. Toth, C. Tubiana, Summer fireworks on comet 67P. Mon. Not. R. Astron. Soc. 462, S184-S194 (2016). https://doi.org/10.1093/mnras/stw2409. arXiv: 1609.07743

J.B. Vincent, S.F. Hviid, S. Mottola, E. Kuehrt, F. Preusker, F. Scholten, H.U. Keller, N. Oklay, D. de Niem, B. Davidsson, M. Fulle, M. Pajola, M. Hofmann, X. Hu, H. Rickman, Z.Y. Lin, C. Feller, A. Gicquel, S. Boudreault, H. Sierks, C. Barbieri, P.L. Lamy, R. Rodrigo, D. Koschny, M.F. A'Hearn, M.A. Barucci, J.L. Bertaux, I. Bertini, G. Cremonese, V. Da Deppo, S. Debei, M. De Cecco, J. Deller, S. Fornasier, O. Groussin, P.J. Gutiérrez, P. Gutiérrez-Marquez, C. Güttler, W.H. Ip, L. Jorda, J. Knollenberg, G. Kovacs, J.R. Kramm, M. Küppers, L.M. Lara, M. Lazzarin, J.J. Lopez Moreno, F. Marzari, G. Naletto, L. Penasa, X. Shi, N. Thomas, I. Toth, C. Tubiana, Constraints on cometary surface evolution derived from a statistical analysis of 67P's topography. Mon. Not. R. Astron. Soc. 469, S329-S338 (2017). https://doi.org/10.1093/mnras/stx1691. arXiv:1707.00734

Z. Yoldi, A. Pommerol, B. Jost, O. Poch, J. Gouman, N. Thomas, VIS-NIR reflectance of water ice/regolith analogue mixtures and implications for the detectability of ice mixed within planetary regoliths. Geophys. Res. Lett. 42, 6205-6212 (2015). https://doi.org/10.1002/2015GL064780 